\title{
DRINFELD MODULES AND TORSION IN THE CHOW GROUPS OF CERTAIN THREEFOLDS
}

\author{
CHAD SCHOEN AND JAAP TOP
}

\begin{abstract}
Let $E \rightarrow B$ be an elliptic surface defined over the algebraic closure of a finite field of characteristic greater than 5. Let $W$ be a resolution of singularities of $E \times_{B} E$. We show that the $l$-adic Abel-Jacobi map from the $l$-power-torsion in the second Chow group of $W$ to $H^{3}\left(W, \mathbb{Z}_{l}(2)\right) \otimes \mathbb{Q}_{l} / \mathbb{Z}_{l}$ is an isomorphism for almost all primes $l$. A main tool in the proof is the assertion that certain CM-cycles in fibres of $W \rightarrow B$ are torsion, which is proven using results from the theory of Drinfeld modular curves.
\end{abstract}

\section{Introduction}

Let $\overline{\mathbb{F}}$ be an algebraic closure of a finite field $\mathbb{F}$ of characteristic $p$. For a smooth projective variety $V / \mathbb{F}$, let $Z_{\text {rat }}^{r}\left(V_{\overline{\mathbb{F}}}\right) \subset Z_{\text {alg }}^{r}\left(V_{\overline{\mathbb{F}}}\right) \subset Z_{\text {hom }}^{r}\left(V_{\overline{\mathbb{F}}}\right)$ denote the groups of codimension $r$ algebraic cycles on $V_{\overline{\mathbb{F}}}$ which are rationally (respectively algebraically, respectively homologically) equivalent to zero. The group $C H_{\mathrm{hom}}^{r}\left(V_{\overline{\mathbb{F}}}\right):=Z_{\mathrm{hom}}^{r}\left(V_{\overline{\mathbb{F}}}\right) / Z_{\mathrm{rat}}^{r}\left(V_{\overline{\mathbb{F}}}\right)$ is a fundamental invariant of $V_{\overline{\mathbb{F}}}$ about which very little is currently known. For each prime number $l$ distinct from $p$ there is an $l$-adic Abel-Jacobi map

$$
\mathbf{a}_{V, l}^{r}: C H_{\mathrm{hom}}^{r}\left(V_{\overline{\mathbb{F}}}\right) \otimes \mathbb{Z}_{l} \longrightarrow H^{2 r-1}\left(V_{\overline{\mathbb{F}}}, \mathbb{Z}_{l}(r)\right) \otimes \mathbb{Q}_{l} / \mathbb{Z}_{l} .
$$

In the cases $r=1$ and $r=\operatorname{dim}(V)$, one knows that $\mathbf{a}_{V, l}^{r}$ is an isomorphism. It is interesting to ask the following.

Question 0.1. Is $\mathbf{a}_{V, l}^{r}$ an isomorphism for all $r$ and all $l \neq p$ ?

In this paper we consider the map $\alpha_{V, l}^{r}$ obtained by restricting $\mathbf{a}_{V, l}^{r}$ to the torsion subgroup of $C H_{\text {hom }}^{r}\left(V_{\overline{\mathbb{F}}}\right) \otimes \mathbb{Z}_{l}$. Note that $C H^{r}\left(V_{\overline{\mathbb{F}}}\right)_{\text {tors }} \otimes \mathbb{Z}_{l}$ is in fact a subgroup of $C H_{\text {hom }}^{r}\left(V_{\overline{\mathbb{F}}}\right) \otimes \mathbb{Z}_{l}$ whenever $H^{2 r}\left(V_{\overline{\mathbb{F}}}, \mathbb{Z}_{l}(r)\right)$ is torsion free which occurs for almost all $l$ [13]. Quite generally, $\alpha_{V, l}^{2}$ is injective (cf. $\S 1$ ). The main purpose of this paper is to show that $\alpha_{V, l}^{2}$ is surjective for almost all $l$ for a particular class of threefolds which we now describe.

Let $B$ be a smooth, geometrically irreducible, projective curve over $\mathbb{F}$. Let $\varpi: E \rightarrow B$ be a non-isotrivial elliptic surface. Suppose that $V$ is a smooth projective variety which is birational to $E \times{ }_{B} E$. If $\varpi$ is semi-stable, we may construct such a $V$ by blowing up the reduced singular locus of $E \times{ }_{B} E$. In order to be able to deal with the non-semi-stable case as well, we assume that $\operatorname{char}(\mathbb{F})>5$. Now $V$ exists by a general theorem on resolution of singularities [1].

Theorem 0.2. For all except possibly finitely many primes $l$, the $l$-adic Abel-Jacobi map gives rise to an isomorphism

$$
\alpha_{V, l}^{2}: C H^{2}\left(V_{\overline{\mathbb{F}}}\right)_{\text {tors }} \otimes \mathbb{Z}_{l} \longrightarrow H^{3}\left(V_{\overline{\mathbb{F}}}, \mathbb{Z}_{l}(2)\right) \otimes \mathbb{Q}_{l} / \mathbb{Z}_{l} .
$$

Received 13 September 2005; revised 19 December 2006; published online 21 June 2007.

2000 Mathematics Subject Classification 14C25 (primary), 11G09, 11G16 (secondary).

The first author gratefully acknowledges support from NSA (MDA904-97-1-0041), NSF (DMS-9306733, DMS9970500, DMS-0200012), and a Duke University Planning Grant for International Research. 
We now give a brief outline of the proof of Theorem 0.2 together with an overview of the contents of each section of the paper.

The first section is devoted to the injectivity of $\alpha_{V, l}^{2}$. This is established by relating $\alpha_{V, l}^{2}$ to Bloch's cycle class map on torsion cycles, which is known to be injective for cycles of codimension 2 .

To prove the surjectivity of $\alpha_{V, l}^{2}$, it is shown in Section 2 that it suffices to treat the case in which the elliptic surface $\varpi$ is semi-stable. In this case there is an explicit subgroup

$$
C H_{\mathrm{CM}}^{2}\left(V_{\overline{\mathbb{F}}}\right) \subset C H_{\text {hom }}^{2}\left(V_{\overline{\mathbb{F}}}\right),
$$

generated by so-called complex multiplication cycles, with the property that the Abel-Jacobi map restricted to $C H_{\mathrm{CM}}^{2}\left(V_{\overline{\mathbb{F}}}\right)+C H_{\mathrm{alg}}^{2}\left(V_{\overline{\mathbb{F}}}\right)$ is surjective for almost all primes $l[\mathbf{2 6}, \S 11]$. Since $C H_{\mathrm{alg}}^{2}\left(V_{\overline{\mathbb{F}}}\right)$ is a torsion group, the theorem will be proved if one can show that complex multiplication $(\mathrm{CM})$ cycles lie in $C H^{2}\left(V_{\overline{\mathbb{F}}}\right)_{\text {tors }}$. Complex multiplication cycles live in the open subvariety $\dot{V}_{\overline{\mathbb{F}}} \subset V_{\overline{\mathbb{F}}}$, obtained by removing the singular fibers of the map $V_{\overline{\mathbb{F}}} \rightarrow B_{\overline{\mathbb{F}}}$.

In Section 3 it is shown that it suffices to prove that CM cycles live in $C H^{2}\left(\dot{V}_{\overline{\mathbb{F}}}\right)_{\text {tors }}$. The problem of verifying this is a function-field analog of a problem studied by Flach [11] and Mildenhall $[\mathbf{2 3}]$ in the context of elliptic curves over $\mathbb{Q}$. In order to adapt these techniques to our situation we must replace moduli schemes for elliptic curves with Drinfeld modular curves.

Thus Section 4 recalls briefly the notions of Drinfeld module, full level structure, and $\Gamma_{0}$-level structure. Essential facts about the modular varieties for full level structure and their compactifications are recalled in Section 5. Compactified coarse moduli spaces for $\Gamma_{0}$-level structure appear as quotients of the compactified full level structure varieties in Section 6 . Key components of the method of Flach and Mildenhall are the Manin-Drinfeld Theorem, Atkin-Lehner automorphisms and a detailed understanding of the singular fibers in the moduli schemes for a $\Gamma_{0}$-level structure. These topics are treated briefly in Sections 7, 8 and 9 . The fact that an elliptic surface of conductor $x_{\infty} \mathfrak{c}$ with split multiplicative reduction at the point $x_{\infty}$ is dominated by a Drinfeld modular variety for a $\Gamma_{0}(\mathfrak{c})$-level structure is recalled in Section 10.

With these tools in hand one can now proceed, in the final section, to prove the theorem: roughly speaking, representatives $\mathfrak{z} \in Z^{2}\left(\dot{V}_{\overline{\mathbb{F}}}\right)$ of multiples of generators of $C H_{\mathrm{CM}}^{2}\left(V_{\overline{\mathbb{F}}}\right)$ are shown to be supported on the images of certain Drinfeld modular varieties. With the help of the Manin-Drinfeld theorem, rational functions on these modular varieties are constructed whose divisors give rise to multiples of the cycles $\mathfrak{z}$. This shows that CM cycles give torsion elements in $C H^{2}\left(\dot{V}_{\overline{\mathbb{F}}}\right)$ and completes the proof.

We now describe briefly how our result relates to Beilinson's conjecture $[\mathbf{2}, 1.0]$, which states that

$$
C H_{\text {hom }}^{r}\left(V_{\overline{\mathbb{F}}}\right) \subset C H^{r}\left(V_{\overline{\mathbb{F}}}\right)_{\text {tors }}
$$

for any smooth projective variety over the algebraic closure of a finite field. Recall that this conjecture is known to hold for products of three curves over $\mathbb{F}$ and closely related varieties [31]. Here we are interested in the case that $V$ is birational to $E \times{ }_{B} E$ and the tautological rational map $f: V \rightarrow B$ is a morphism. In this case there is an exact localization sequence,

$$
\bigoplus_{b \in\left|B_{\overline{\mathbb{F}}}\right|_{0}} C H^{1}\left(f^{-1}(b)\right) \stackrel{\oplus i_{b *}}{\longrightarrow} C H^{2}\left(V_{\overline{\mathbb{F}}}\right) \stackrel{u}{\longrightarrow} C H^{2}\left(E_{\eta} \times_{\eta} E_{\eta}\right) \longrightarrow 0,
$$

where $\eta \in B_{\overline{\mathbb{F}}}$ is the generic point. Our results imply that $\operatorname{im}\left(\bigoplus i_{b *}\right) \cap C H_{\text {hom }}^{2}\left(V_{\overline{\mathbb{F}}}\right)$ is a torsion group plus a finitely generated free abelian group. The free summand is generated by cycles in the supersingular fibers and singular fibers where the reduction is not semi-stable. We do not know whether this summand is zero or not. The problem of showing that the group $u\left(C H_{\text {hom }}^{2}\left(V_{\overline{\mathbb{F}}}\right)\right)$ is torsion appears to be closely related to a function field analog of the following well-known and difficult conjecture. 
Conjecture 0.3 (Beilinson [2, 5.1], Bloch [4, Introduction]). Let $S$ be a smooth, irreducible, $d$-dimensional variety defined over a number field. Then the albanese map,

$$
C H_{\mathrm{hom}}^{d}\left(S_{\overline{\mathbb{Q}}}\right) \longrightarrow \operatorname{Alb}_{S}(\overline{\mathbb{Q}}),
$$

is an isomorphism.

Acknowledgements. We wish to thank S. Bloch for asking whether complex multiplication cycles are torsion when the base field is finite. We are grateful to E. U. Gekeler for establishing a form of the Manin-Drinfeld theorem suitable for the present context [15]. It is furthermore a pleasure to thank Marius van der Put, Gert-Jan van der Heiden and Lenny Taelman for helpful conversations concerning Drinfeld modular schemes.

Notational conventions. Throughout:

$*_{\text {tors }}$ is the torsion subgroup of an abelian group $*$;

$Z^{r}(V)$ is the free abelian group on irreducible codimension $r$ subvarieties of a variety $V$;

$C H^{r}(V)=Z^{r}(V) / Z_{\text {rat }}^{r}(V)$;

$C H_{\text {alg }}^{r}(V)=Z_{\text {alg }}^{r}(V) / Z_{\text {rat }}^{r}(V)$;

$Z_{\text {hom }}^{r}(V):=\operatorname{Ker}\left[Z^{r}(V) \rightarrow \prod_{l \neq \operatorname{char}(F)} H^{2 r}\left(V_{\bar{F}}, \mathbb{Z}_{l}(r)\right)\right]$, where $V$ is smooth over a field $F$ with algebraic closure $\bar{F}$;

$\mathbb{F}$ is a finite field of characteristic $p$.

In all sections except Section 1:

$X$ is a smooth, projective, geometrically connected curve;

$\pi: Y \rightarrow X$ is a regular, relatively minimal, non-isotrivial elliptic surface with section;

$W$ is the blow-up of $Y \times_{X} Y$ along the reduced singular locus.

Abuse of notation: if $c: P \rightarrow Q$ is a morphism of schemes over a field $k$, and $k \subset k^{\prime}$ is a field extension, then the base changed morphism $c_{k^{\prime}}: P_{k^{\prime}} \rightarrow Q_{k^{\prime}}$ will frequently be denoted $c$, without the subscript $k^{\prime}$.

\section{Injectivity of the l-adic Abel-Jacobi map}

Let $U$ be a smooth, projective variety over a field $F$ which is finitely generated over its prime subfield. Let $\bar{F}$ be a separable closure of $F$. The purpose of this section is to prove the following result.

Proposition 1.1. For any prime $l \neq \operatorname{char}(F)$ the restriction of $\mathbf{a}_{U, l}^{2}$ to $C H_{\text {hom }}^{2}\left(U_{\bar{F}}\right)_{\text {tors }} \otimes \mathbb{Z}_{l}$ is injective.

Proof. The $l$-adic Abel-Jacobi map $\mathbf{a}_{U, l}^{r}$ may be defined in terms of extensions of Galois modules or in terms of the ordinary cycle class map to $H^{2 r}\left(U_{F^{\prime}}, \mathbb{Z}_{l}(r)\right)$ for $\left[F^{\prime}: F\right]<\infty$ by means of the Hochschild-Serre spectral sequence $[19, \S 9]$. To reconcile the form given below with the $l$-adic Abel-Jacobi map mentioned in the introduction, see Remark 1.4. In general the Abel-Jacobi map takes the form

$$
\mathbf{a}_{U, l}^{r}: C H_{\mathrm{hom}}^{r}\left(U_{\bar{F}}\right) \simeq \lim _{F^{\prime}} C H_{\mathrm{hom}}^{r}\left(U_{F^{\prime}}\right) \longrightarrow \lim _{F^{\prime}} H^{1}\left(G_{F^{\prime}}, \mathbf{H}\right),
$$

where the direct limit is taken over intermediate fields, $F \subset F^{\prime} \subset \bar{F}$, with $\left[F^{\prime}: F\right]<\infty, G_{F^{\prime}}=$ $\operatorname{Gal}\left(\bar{F} / F^{\prime}\right), \mathbf{H}=H^{2 r-1}\left(U_{\bar{F}}, \mathbb{Z}_{l}(r)\right) /$ tors, and group cohomology is taken with continuous cocycles for the $l$-adic topology [34]. The torsion subgroup of the right-most term of (1.1) is canonically identified with

$$
\lim _{F^{\prime}}\left(H^{2 r-1}\left(U_{\bar{F}}, \mathbb{Z}_{l}(r)\right) \otimes \mathbb{Q}_{l} / \mathbb{Z}_{l}\right)^{G_{F^{\prime}}} \simeq H^{2 r-1}\left(U_{\bar{F}}, \mathbb{Z}_{l}(r)\right) \otimes \mathbb{Q}_{l} / \mathbb{Z}_{l}
$$


On the other hand, Bloch has defined a cycle class map [3],

$$
\lambda^{r}: C H^{r}\left(U_{\bar{F}}\right)_{\text {tors }} \otimes \mathbb{Z}_{l} \longrightarrow H^{2 r-1}\left(U_{\bar{F}}, \mathbb{Q}_{l} / \mathbb{Z}_{l}(r)\right),
$$

which is injective when $r=2$ [6, Corollaire 4]. As the right-hand side of (1.2) is naturally identified with the kernel of $H^{2 r-1}\left(U_{\bar{F}}, \mathbb{Q}_{l} / \mathbb{Z}_{l}(r)\right) \rightarrow H^{2 r}\left(U_{\bar{F}}, \mathbb{Z}_{l}(r)\right)$, the proposition follows from the following result.

TheOREM 1.2. The restrictions of $\lambda^{r}$ and $\mathbf{a}_{U, l}^{r}$ to $C H_{\text {hom }}^{r}\left(U_{\bar{F}}\right)_{\text {tors }} \otimes \mathbb{Z}_{l}$ agree up to sign.

Proof. Let $\mathcal{H}^{r}\left(\mathbb{Q}_{l} / \mathbb{Z}_{l}(r)\right)$ denote the Zariski sheaf associated to the presheaf whose value on an open $V \subset U_{F^{\prime}}$ is the group $H_{\mathrm{et}}^{r}\left(V, \mathbb{Q}_{l} / \mathbb{Z}_{l}(r)\right)$. Consider the diagram

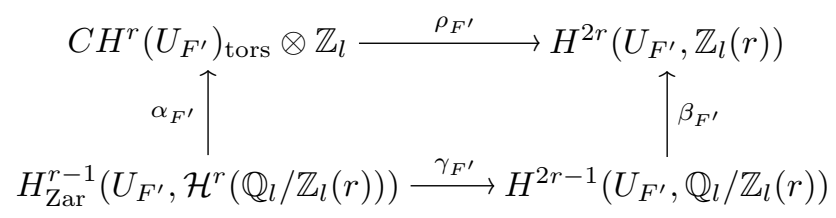

which is commutative up to sign; see [6, Corollaire 1] or [5, 3.8]. We refer to [6] or [5] for the definitions of the maps and the fact that $\alpha_{F^{\prime}}$ is surjective. Bloch's cycle class map is the unique map,

$$
\lambda^{r}: C H^{r}\left(U_{\bar{F}}\right)_{\text {tors }} \otimes \mathbb{Z}_{l} \longrightarrow H^{2 r-1}\left(U_{\bar{F}}, \mathbb{Q}_{l} / \mathbb{Z}_{l}(r)\right),
$$

satisfying $\lambda^{r} \circ \lim _{F^{\prime}} \alpha_{F^{\prime}}=\lim _{F^{\prime}} \gamma_{F^{\prime}}$; see [6, Corollaire 4] or [5, 4.3; 24, III.1.16]. Define

$$
\begin{aligned}
D_{F^{\prime}} & =\operatorname{Ker}\left[H^{2 r}\left(U_{F^{\prime}}, \mathbb{Z}_{l}(r)\right) \stackrel{f_{F^{\prime}}}{\longrightarrow} H^{2 r}\left(U_{\bar{F}}, \mathbb{Z}_{l}(r)\right)\right], \\
E_{F^{\prime}} & =\operatorname{Ker}\left[f_{F^{\prime}} \circ \beta_{F^{\prime}}: H^{2 r-1}\left(U_{F^{\prime}}, \mathbb{Q}_{l} / \mathbb{Z}_{l}(r)\right) \rightarrow H^{2 r}\left(U_{\bar{F}}, \mathbb{Z}_{l}(r)\right)\right], \\
H_{F^{\prime}} & =\operatorname{Ker}\left(f_{F^{\prime}} \circ \beta_{F^{\prime}} \circ \gamma_{F^{\prime}}\right) .
\end{aligned}
$$

Now (1.4) gives rise to the left-hand square in

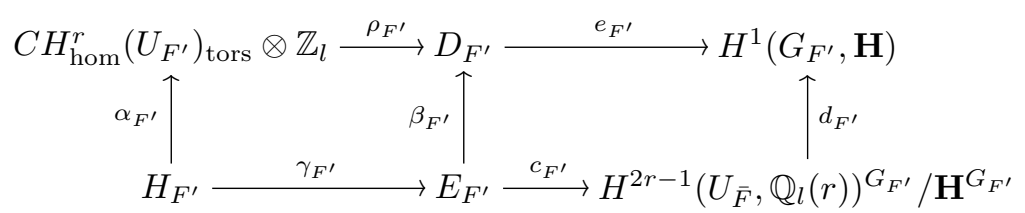

The map $e_{F^{\prime}}$ arises from the Hochschild-Serre spectral sequence for cohomology with $\mu_{l^{m}}^{\otimes r}$ coefficients by taking the projective limit with respect to $m$. The map $\lim _{F^{\prime}} e_{F^{\prime}} \circ \rho_{F^{\prime}}$ is the restriction of the $l$-adic Abel-Jacobi map to $C H_{\text {hom }}^{r}\left(U_{\bar{F}}\right)_{\text {tors }} \otimes \mathbb{Z}_{l}$. On the other hand, the restriction of $\lambda^{r}$ to $C H_{\text {hom }}^{r}\left(U_{\bar{F}}\right)_{\text {tors }} \otimes \mathbb{Z}_{l}$ factors through $\lim _{F^{\prime}} c_{F^{\prime}}$. Thus the agreement up to sign of the restrictions of $\mathbf{a}_{U, l}^{2}$ and $\lambda^{r}$ will follow from the commutativity of the right-hand square in (1.5).

To establish this commutativity we work with cohomology with finite coefficients. Associated to the short exact sequence

$$
1 \longrightarrow \mu_{l^{m}}^{\otimes r} \longrightarrow \mu_{l^{n+m}}^{\otimes r} \longrightarrow \mu_{l^{n}}^{\otimes r} \longrightarrow 1
$$

are the Bockstein (coboundary) maps

$$
\beta_{F^{\prime}}^{i}(n, m): H^{i}\left(U_{F^{\prime}}, \mu_{l^{n}}^{\otimes r}\right) \longrightarrow H^{i+1}\left(U_{F^{\prime}}, \mu_{l^{m}}^{\otimes r}\right),
$$

analogous maps $\beta_{\bar{F}}^{i}(n, m)$ with $\bar{F}$ replacing $F^{\prime}$ in (1.7), and the Galois coboundary map

$$
\delta(n, m): \operatorname{Ker}\left(\beta_{\bar{F}}^{2 r-1}(n, m)\right)^{G_{F^{\prime}}} \longrightarrow \mathrm{H}^{1}\left(G_{F^{\prime}}, \operatorname{Coker}\left(\beta_{\bar{F}}^{2 r-2}(n, m)\right)\right),
$$


coming from the short exact sequence of $G_{F^{\prime}}$-modules

$$
0 \longrightarrow \operatorname{Coker}\left(\beta_{\bar{F}}^{2 r-2}(n, m)\right) \longrightarrow H^{2 r-1}\left(U_{\bar{F}}, \mu_{l^{n+m}}^{\otimes r}\right) \longrightarrow \operatorname{Ker}\left(\beta_{\bar{F}}^{2 r-1}(n, m)\right) \longrightarrow 0 .
$$

Define

$$
\begin{aligned}
L^{1} H^{2 r}\left(U_{F^{\prime}}, \mu_{l^{m}}^{\otimes r}\right) & =\operatorname{Ker}\left[H^{2 r}\left(U_{F^{\prime}}, \mu_{l^{m}}^{\otimes r}\right) \stackrel{s}{\longrightarrow} H^{2 r}\left(U_{\bar{F}}, \mu_{l^{m}}^{\otimes r}\right)\right], \\
H^{2 r-1}\left(U_{F^{\prime}}, \mu_{l^{n}}^{\otimes r}\right)_{0} & =\operatorname{Ker}\left(s \circ \beta_{F^{\prime}}^{2 r-1}(n, m)\right), \\
\mathbf{H}_{m n} & =\operatorname{Coker}\left(\beta_{\bar{F}}^{2 r-2}(n, m)\right), \\
\mathbf{K}_{m n} & =\operatorname{Ker}\left(\beta_{\bar{F}}^{2 r-1}(n, m)\right) .
\end{aligned}
$$

Now the commutativity of the right-hand square in (1.5) may be deduced from the following lemma by applying an inverse limit in $m$ and a direct limit in $n$.

LEMma 1.3. The following diagram commutes:

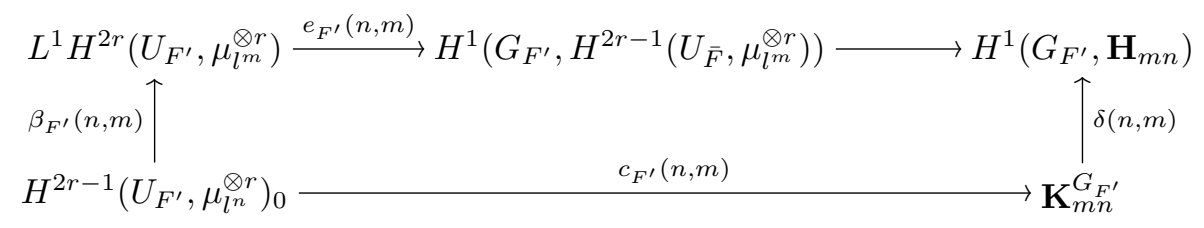

where $e_{F^{\prime}}(n, m)$ comes from the Hochschild-Serre spectral sequence.

Proof. A similar commutativity result is proved in [19, 9.5]. We reduce the current problem to the one treated there.

Take an injective resolution of (1.6) and apply the functor $\varrho_{*}$, where $\varrho: U_{F^{\prime}} \rightarrow \operatorname{Spec}\left(F^{\prime}\right)$ is the structure morphism. This gives an exact sequence of complexes of injective sheaves on $\operatorname{Spec}\left(F^{\prime}\right)[\mathbf{2 4}$, III.1.2] which forms the first row in the following commutative diagram with exact rows $[36,1.5]$ :

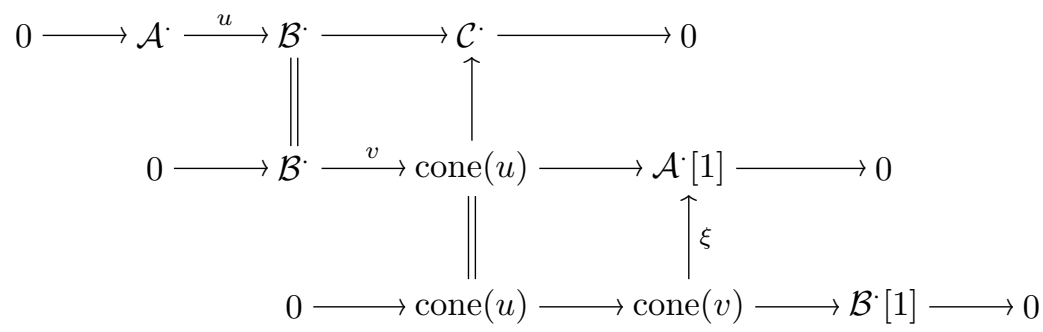

Let $f$ : Sheaves on $\operatorname{Spec}\left(F^{\prime}\right) \rightarrow \mathbf{A b}$ denote the global section functor. The Grothendieck spectral sequence for the composite functor $f \circ \varrho_{*}$ may be identified with the Hochschild-Serre spectral sequence: the category of etale sheaves of abelian groups on $\operatorname{Spec}\left(F^{\prime}\right)$ is equivalent to the category of discrete $G_{F^{\prime}}$-modules and $f$ may be identified with the functor which associates to a $G_{F^{\prime}}$-module its $G_{F^{\prime}}$-invariant subgroup. In this context the long exact sequence of cohomology groups $H^{\bullet}\left(U_{\overline{\mathbb{F}}}, \mu_{l}^{\otimes}\right)$ associated with (1.6) is identified with the long exact sequence of etale sheaves on $\operatorname{Spec}\left(F^{\prime}\right)$,

$$
\cdots \longrightarrow \mathcal{H}^{n} \mathcal{A} \longrightarrow \mathcal{H}^{n} \mathcal{B} \cdot \mathcal{H}^{n} \mathcal{C} \longrightarrow \mathcal{H}^{n+1} \mathcal{A} \longrightarrow \cdots
$$

By $[\mathbf{3 6}, 1.5]$ this is isomorphic to the cohomology sequence

$$
\longrightarrow \mathcal{H}^{n-1}(\operatorname{cone}(v)) \stackrel{\psi^{n-1}}{\longrightarrow} \mathcal{H}^{n-1}(\mathcal{B} \cdot[1]) \stackrel{\partial^{n-1}}{\longrightarrow} \mathcal{H}^{n}(\operatorname{cone}(u)) \stackrel{\mu^{n}}{\longrightarrow} \mathcal{H}^{n}(\operatorname{cone}(v)) \longrightarrow .
$$


The map $\delta(n, m)$ of Lemma 1.3 is now identified with the coboundary map, $\delta: f Y \rightarrow R^{1} f X$, associated to the short exact sequence with $n=2 r-1$,

$$
X:=\operatorname{im}\left(\psi^{n-1}\right) \hookrightarrow \mathcal{H}^{n-1}\left(\mathcal{B}^{\cdot}[1]\right) \rightarrow \operatorname{im}\left(\partial^{n-1}\right)=\operatorname{Ker}\left(\mu^{n}\right)=: Y .
$$

The cohomology group $H^{2 r-1}\left(U_{F^{\prime}}, \mu_{l^{n}}^{\otimes r}\right)$ is identified with the hypercohomology group $\mathbb{R}^{2 r-1} f \mathcal{C}$. The map $\beta_{F^{\prime}}(n, m)$ in Lemma 1.3 is induced by the map on the hypercohomology $\mathbb{R}^{2 r-1} f \mathcal{C} \rightarrow \mathbb{R}^{2 r} f \mathcal{A}$. Since the objects in (1.9) are $f$-acyclic, hypercohomology is just the cohomology of $f$ applied to each complex. Since cone and $f$ commute, the long exact hypercohomology sequence for the top row in (1.9) is isomorphic to that for the bottom row. Thus $\beta_{F^{\prime}}(n, m)$ comes from the natural map $\nu: \mathbb{R}^{2 r-1} f($ cone $(u)) \rightarrow \mathbb{R}^{2 r-1} f(\operatorname{cone}(v))$.

To go further we need the hypercohomology spectral sequence on $\mathbb{R}^{n} f \mathcal{A}$ which may be constructed as follows. Endow $\mathcal{A}$ with the canonical filtration $[\mathbf{7}, 1.4 .6]$, and let $\mathcal{A} \rightarrow \mathcal{J}$. be a filtered quasi-isomorphism to a filtered complex whose associated graded complexes are $f$-acyclic. Then the spectral sequence of the filtered complex $f \mathcal{J}$ is the hypercohomology spectral sequence up to a shift of indices [7, 1.4]. Since $\xi$ is a filtered quasi-isomorphism when both complexes are given the canonical filtration, the hypercohomology spectral sequences for the functor $f$ and the complexes $\mathcal{A}^{\cdot}[1]$ and cone $(v)$ are isomorphic. Consider the commutative diagram with $n=2 r-1$,

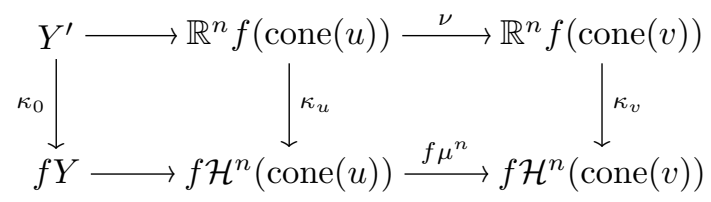

where $\kappa_{u}$ and $\kappa_{v}$ are standard maps from the spectral sequences $Y^{\prime}:=\operatorname{Ker}\left(\kappa_{v} \circ \nu\right)$ and $f Y \simeq \operatorname{Ker}\left(f \mu^{n}\right)$. Denote the filtration on the hypercohomology $\mathbb{R}^{n} f(\operatorname{cone}(v))$ coming from the hypercohomology spectral sequence by $F^{\bullet}$. Now the diagram in Lemma 1.3 is written in the current notation as

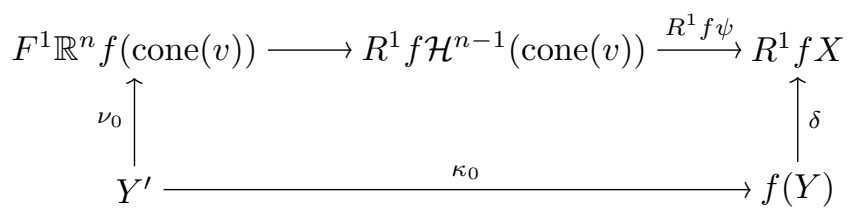

where $\nu_{0}$ is induced by $\nu$. This diagram commutes by $[\mathbf{1 9}, 9.5]$ applied to the bottom row in (1.9).

This completes the proof of Theorem 1.2 and of Proposition 1.1.

Remark 1.4. To relate the Abel-Jacobi maps (0.1) and (1.1) observe that when $F^{\prime}$ is a finite field,

$$
H^{1}\left(G_{F^{\prime}}, \mathbf{H}\right) \otimes \mathbb{Q}_{l} \simeq \mathbf{H} \otimes \mathbb{Q}_{l} /\left(1-\operatorname{Frob}_{F^{\prime}}\right)=0,
$$

since by the Weil conjectures (Deligne's theorem) 1 is not an eigenvalue of the action of Frobenius on $\mathbf{H}$. Thus $H^{1}\left(G_{F^{\prime}}, \mathbf{H}\right)$ gets identified with its torsion subgroup which is naturally identified with $\left(\mathbf{H} \otimes \mathbb{Q}_{l} / \mathbb{Z}_{l}\right)^{G_{F^{\prime}}}$.

REMARK 1.5. The cycle class map

$$
\lambda^{r}: C H^{r}\left(U_{\bar{F}}\right)_{\text {tors }} \otimes \mathbb{Z}_{l} \longrightarrow H^{2 r-1}\left(U_{\bar{F}}, \mathbb{Q}_{l} / \mathbb{Z}_{l}(r)\right)
$$

may fail to be injective for $2<r<\operatorname{dim}\left(U_{\bar{F}}\right)$. So far this phenomenon has only been noted when $\operatorname{char}(F)=0$. In light of Question 0.1 it would be interesting to understand the specialization 
of $\operatorname{Ker}\left(\lambda^{r}\right)$ from $F$ to the algebraic closure of a finite field. In this context the cycles in $[\mathbf{3 5}, 7.2]$ seem especially interesting. (See also $[\mathbf{2 7}]$.)

\section{Reduction to the semi-stable case}

With the injectivity of the map $\alpha_{V, l}^{2}$ established, Theorem 0.2 will follow once surjectivity for almost all $l$ is known. The purpose of this section is to show that surjectivity will hold for fiber products of general non-isotrivial elliptic surfaces with section if it holds for fiber products of semi-stable ones.

Let $\varpi: E \rightarrow B$ be a non-isotrivial elliptic surface over a finite field, which we will assume to have characteristic $p>5$. After replacing this field with a finite extension denoted $\mathbb{F}$, we see that the theory of semi-stable reduction [30, VII.5.4] gives a smooth, projective geometrically irreducible curve $X / \mathbb{F}$, and a regular, relatively minimal, non-isotrivial, semi-stable elliptic surface with section $\pi: Y \rightarrow X$, which fits into a commutative diagram

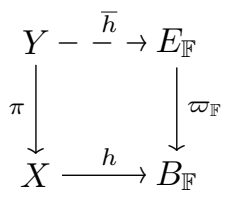

where $h$ is a morphism and $\bar{h}$ is a dominant rational map. Since $\varpi_{\mathbb{F}}$ is non-isotrivial, $Y$ will have at least one singular fiber. The only singular points of $Y \times_{X} Y$ are ordinary double points at points $\left(y_{1}, y_{2}\right)$, where each $y_{i}$ is a singular point of the fiber $\pi^{-1}\left(\pi\left(y_{i}\right)\right)$. These singularities are resolved by a single blow-up of the reduced singular locus. We denote this blow-up by $\sigma: W \rightarrow Y \times_{X} Y$. For $V$ as in Theorem 0.2, the map $\bar{h}$ gives rise to a dominant rational map $\hat{h}: W \rightarrow V_{\mathbb{F}}$. By Abhyankar's theorem [1, p. 1, Dominance], which applies since char $(\mathbb{F})>5$, $\hat{h}_{\overline{\mathbb{F}}}$ factors as $\tilde{h} \circ \kappa^{-1}$,

$$
W_{\overline{\mathbb{F}}} \stackrel{\kappa}{\longleftarrow} \tilde{W}_{\overline{\mathbb{F}}} \stackrel{\tilde{h}}{\longrightarrow} V_{\overline{\mathbb{F}}},
$$

where $\kappa$ is a sequence of blow-ups with smooth centers and $\tilde{h}$ is a surjective morphism.

LEMma 2.1. (i) The map $\alpha_{W, l}^{2}$ is surjective if and only if $\alpha_{\tilde{W}, l}^{2}$ is.

(ii) If $\alpha_{\tilde{W}, l}^{2}$ is surjective, then so is $\alpha_{V, l}^{2}$.

Proof. (i) Blowing up a point changes neither the domain nor the target of $\alpha_{l}^{2}$. Blowing up a smooth curve $C$ adds a direct summand $C H^{1}\left(C_{\overline{\mathbb{F}}}\right)_{\text {tors }} \otimes \mathbb{Z}_{l}$ to the domain of $\alpha_{l}^{2}$ and a summand $H^{1}\left(C_{\overline{\mathbb{F}}}, \mathbb{Z}_{l}(1)\right) \otimes \mathbb{Q}_{l} / \mathbb{Z}_{l}$ to the target. The cycle class map on the blow-up gives rise to the Kummer theory isomorphism between these two groups [12,6.7(d) and 3.3(b)].

(ii) By the projection formula the map

$$
\tilde{h}_{*}: H^{3}\left(\tilde{W}_{\overline{\mathbb{F}}}, \mathbb{Z}_{l}(2)\right) \otimes \mathbb{Q}_{l} / \mathbb{Z}_{l} \longrightarrow H^{3}\left(V_{\overline{\mathbb{F}}}, \mathbb{Z}_{l}(2)\right) \otimes \mathbb{Q}_{l} / \mathbb{Z}_{l}
$$

is surjective. The assertion follows from the functoriality of the Abel-Jacobi map with respect to a proper direct image $[\mathbf{3}, \S 3 ; \mathbf{2 5}, 1.10]$.

\section{Reduction to a theorem about complex multiplication cycles}

In this section we recall the notion of complex multiplication (CM) cycle and reduce Theorem 0.2 to an assertion about CM cycles.

Let $\pi: Y \rightarrow X$ denote a semi-stable, non-isotrivial elliptic surface with section over a finite field $\mathbb{F}$. Recall that $W$ is the blow-up of the fiber product along the reduced singular locus. The inclusion of the locus where $\pi: Y \rightarrow X$ is smooth will be denoted $j: \dot{X} \rightarrow X$ and base 
change by $j$ will be indicated by adding a dot, ${ }^{\cdot}$, to the notation. Define $\Xi:=X-\dot{X}$. This set is non-empty since $\pi$ is not isotrivial. Let $f: W \rightarrow X$ be the tautological map.

There are only finitely many points $\bar{x} \in \dot{X}_{\overline{\mathbb{F}}}$ for which $\pi^{-1}(\bar{x})$ is a supersingular elliptic curve $[30$, V.4]. The remaining closed points will be called complex multiplication (CM) points. Let $\bar{x} \in \dot{X}_{\overline{\mathrm{F}}}$ be a CM point. Then $\operatorname{End}\left(\pi^{-1}(\bar{x})\right)$ is an order in an imaginary quadratic number field and the Néron-Severi group $N^{1}\left(f^{-1}(\bar{x})\right)$ is a free $\mathbb{Z}$-module of rank 4. Write $\Delta_{\bar{x}} \subset f^{-1}(\bar{x}) \simeq$ $\pi^{-1}(\bar{x}) \times \pi^{-1}(\bar{x})$ for the diagonal and $N_{0}^{1}\left(f^{-1}(\bar{x})\right) \subset N^{1}\left(f^{-1}(\bar{x})\right)$ for the rank 3 submodule spanned by $\left\{\pi^{-1}(\bar{x}) \times s(\bar{x}), s(\bar{x}) \times \pi^{-1}(\bar{x}), \Delta_{\bar{x}}\right\}$, where $s: X \rightarrow Y$ is a section of $\pi$.

Definition 3.1. Let $\bar{x} \in \dot{X}_{\overline{\mathbb{F}}}$ be a CM point. A 1-dimensional cycle $\mathfrak{z}$ supported in the fiber $f^{-1}(\bar{x})$ is called a complex multiplication (CM) cycle if the class of $\mathfrak{z}$ in $N^{1}\left(f^{-1}(\bar{x})\right)$ generates the free $\mathbb{Z}$-module $N_{0}^{1}\left(f^{-1}(\bar{x})\right)^{\perp}$ of rank 1 .

The subgroup of $C H^{2}\left(W_{\overline{\mathbb{F}}}\right)$ generated by the classes of all CM cycles in $Z^{1}\left(f^{-1}(\bar{x})\right)$ as $\bar{x}$ ranges over all $\mathrm{CM}$ points will be denoted $C H_{\mathrm{CM}}^{2}\left(W_{\overline{\mathbb{F}}}\right)$.

Lemma 3.2. $C H_{\mathrm{CM}}^{2}\left(W_{\overline{\mathbb{F}}}\right) \subset C H_{\mathrm{hom}}^{2}\left(W_{\overline{\mathbb{F}}}\right)$.

Proof. From the non-isotriviality of $\pi$ and the fact that complex multiplication cycles annihilate $N_{0}^{1}\left(f^{-1}(\bar{x})\right)$ one deduces that their cohomology classes annihilate $H^{2}\left(W_{\overline{\mathbb{F}}}, \mathbb{Q}_{l}(1)\right)$ under the cup product pairing $[\mathbf{2 5}, 5.4]$. Since $H^{4}\left(W_{\overline{\mathbb{F}}}, \mathbb{Z}_{l}(2)\right)$ is torsion free for each $l \neq \operatorname{char}(\mathbb{F})$ $[\mathbf{2 8}, 8.7(\mathrm{i})]$, complex multiplication cycles are homologous to zero.

Write $m_{\pi}$ for the least common multiple of all $n$ for which $\pi$ has a singular fiber of Kodaira type $\mathrm{I}_{n}$. As mentioned in the introduction, the proof of Theorem 0.2 makes use of the following.

Proposition 3.3. If $l \nmid 2 \cdot 5 \cdot p \cdot m_{\pi}$, then

$$
\mathbf{a}_{W, l}^{2}\left(C H_{\mathrm{alg}}^{2}\left(W_{\overline{\mathbb{F}}}\right)+C H_{\mathrm{CM}}^{2}\left(W_{\overline{\mathbb{F}}}\right)\right)=H^{3}\left(W_{\overline{\mathbb{F}}}, \mathbb{Z}_{l}(2)\right) \otimes \mathbb{Q}_{l} / \mathbb{Z}_{l} .
$$

Proof. See $[\mathbf{2 6}, 11.3 .2]$.

Let $U$ be a smooth, projective variety over a field $F$ with algebraic closure $\bar{F}$. An additional ingredient in the proof of Theorem 0.2 is the following well-known fact.

Proposition 3.4. If $F$ is a finite field, then $C H_{\text {alg }}^{r}\left(U_{\bar{F}}\right)$ is a torsion group.

Proof. It follows from the definition of algebraic equivalence, that $C H_{\text {alg }}^{r}\left(U_{\bar{F}}\right)$ is isomorphic to a quotient of $\bigoplus_{C} \operatorname{Jac}(C)(\bar{F})$, where the sum is over a (possibly infinite) collection of curves. When $\bar{F}$ is the algebraic closure of a finite field, the $\bar{F}$-rational points in any abelian variety form a torsion group.

Write $\dot{W} \subset W$ for the complement of the singular fibers. Using techniques from the theory of Drinfeld modules we will show in subsequent sections that complex multiplication cycles give torsion elements in $C H^{2}\left(\dot{W}_{\overline{\mathbb{F}}}\right)$. In order to conclude that complex multiplication cycles are torsion in $C H^{2}\left(W_{\overline{\mathbb{F}}}\right)$ we must study the exact localization sequence

$$
C H^{1}\left(f^{-1}\left(\Xi_{\overline{\mathbb{F}}}\right)\right) \longrightarrow C H^{2}\left(W_{\overline{\mathbb{F}}}\right) \stackrel{\tilde{j}^{*}}{\longrightarrow} C H^{2}\left(\dot{W}_{\overline{\mathbb{F}}}\right) \longrightarrow 0 .
$$

The result we need is the following.

Proposition 3.5. The kernel of the restriction of $\tilde{j}^{*}$ to $C H_{\mathrm{hom}}^{2}\left(W_{\overline{\mathbb{F}}}\right)$ is a torsion group. 
Proof. By the localization sequence, one is reduced to proving that if $z \in Z_{\mathrm{hom}}^{2}\left(W_{\overline{\mathbb{F}}}\right)$ is supported on $f^{-1}\left(\Xi_{\overline{\mathbb{F}}}\right)$ then the class of $z$ in $C H^{2}\left(W_{\overline{\mathbb{F}}}\right)$ is torsion.

Write $z=\sum_{\xi \in \Xi_{\overline{\mathbb{F}}}} z_{\xi}$ with $z_{\xi} \in Z^{1}\left(f^{-1}(\xi)\right)$. Write $s: X \rightarrow Y$ for the zero section. Define divisors $T_{1}=Y \times_{X} s(X)$ and $T_{2}=s(X) \times_{X} Y$ on $Y \times_{X} Y$ minus the singular locus. We will view these as divisors on $W$. Let $T_{3} \subset W$ be the strict transform of the diagonal in $Y \times_{X} Y$. Write $a_{i, \xi}$ for the intersection number $T_{i} \cdot z_{\xi}$. Define

$$
\begin{aligned}
S_{1} & =-T_{1}+T_{2}+T_{3}, \\
S_{2} & =T_{1}-T_{2}+T_{3}, \\
S_{3} & =T_{1}+T_{2}-T_{3}, \\
z_{\xi}^{\prime} & =2 z_{\xi}-\sum_{1 \leqslant i \leqslant 3} a_{i, \xi} S_{i} \cdot f^{-1}(\xi) .
\end{aligned}
$$

Lemma 3.6. The cycle $z_{\xi}^{\prime}$ is contained in $Z_{\mathrm{hom}}^{2}\left(W_{\overline{\mathbb{F}}}\right)$.

Proof. Since $H^{4}\left(W_{\overline{\mathbb{F}}}, \mathbb{Z}_{l}(2)\right)$ is torsion free for all $l \neq p[\mathbf{2 8}, 8.7(\mathrm{i})]$, it suffices to show that $z_{\xi}^{\prime} \cdot H^{2}\left(W_{\overline{\mathbb{F}}}, \mathbb{Q}_{l}(1)\right)=0$. Write $q_{i}: W \rightarrow Y$ for the composition of $\sigma$ with the projection on the $i$ th factor in the fiber product $Y \times_{X} Y$. It is not difficult to show that $q_{i}^{*}\left(H^{2}\left(Y_{\overline{\mathbb{F}}}, \mathbb{Q}_{l}(1)\right)\right)$, for $i \in\{1,2\}$, together with the cohomology classes of the $T_{i}$ and the components of the fibers generate $H^{2}\left(W_{\overline{\mathbb{F}}}, \mathbb{Q}_{l}(1)\right)[\mathbf{2 8}, 7.1,7.3(\mathrm{ii})$-(iii), 7.9, 8.7(i)]. Define

$$
L H^{2}\left(Y_{\overline{\mathbb{F}}}, \mathbb{Q}_{l}(1)\right):=\operatorname{Ker}\left[H^{2}\left(Y_{\overline{\mathbb{F}}}, \mathbb{Q}_{l}(1)\right) \rightarrow H^{2}\left(\pi^{-1}(\xi), \mathbb{Q}_{l}(1)\right)\right] .
$$

Since the cohomology classes of the components of $\pi^{-1}(\xi)$ together with the cohomology class of the section $s$ generate a subgroup of $H^{2}\left(Y_{\overline{\mathbb{F}}}, \mathbb{Q}_{l}(1)\right)$ which maps surjectively to $H^{2}\left(\pi^{-1}(\xi), \mathbb{Q}_{l}(1)\right)$, one may in fact replace $q_{i}^{*}\left(H^{2}\left(Y_{\overline{\mathbb{F}}}, \mathbb{Q}_{l}(1)\right)\right)$ with $q_{i}^{*}\left(L H^{2}\left(Y_{\overline{\mathbb{F}}}, \mathbb{Q}_{l}(1)\right)\right)$ in the description of a generating set for $H^{2}\left(W_{\overline{\mathbb{F}}}, \mathbb{Q}_{l}(1)\right)$. By the projection formula,

$$
z_{\xi}^{\prime} \cdot q_{i}^{*}\left(L H^{2}\left(Y_{\overline{\mathbb{F}}}, \mathbb{Q}_{l}(1)\right)\right)=0 .
$$

Also $z_{\xi}^{\prime}$ is orthogonal to any fiber component. Finally, $z_{\xi}^{\prime} \cdot T_{j}=0$ for all $j$ because

$$
S_{i} \cdot T_{j} \cdot f^{-1}(\xi)=2 \delta_{i j} .
$$

This proves Lemma 3.6.

Lemma 3.7. The class of $z_{\xi}^{\prime}$ in $C H_{1}\left(f^{-1}(\xi)\right)$ is torsion.

Proof. Recall that $\pi^{-1}(\xi) \subset Y$ is a fiber of Kodaira type $\mathrm{I}_{m}$ for some $m>0$. The fiber $f^{-1}(\xi)$ has $2 m^{2}$ irreducible components: $m^{2}$ of these correspond to irreducible components of $\pi^{-1}(\xi) \times \pi^{-1}(\xi)$. The remaining ones arise as exceptional divisors in $W$ when the $m^{2}$ ordinary double points of $Y \times_{X} Y$ which lie over $\xi$ are blown up.

LEMMA 3.8. $C H_{1}\left(f^{-1}(\xi)\right) \simeq \mathbb{Z}^{2 m^{2}+2} \oplus \mathbb{Z} / m \mathbb{Z}$.

Proof. See [29].

To prove Lemma 3.7, it suffices to show that intersection pairing gives a surjective map,

$$
h: \text { N.S. }\left(W_{\overline{\mathbb{F}}}\right) \otimes \mathbb{Q} \longrightarrow \operatorname{Hom}\left(C H_{1}\left(f^{-1}(\xi)\right), \mathbb{Q}\right) .
$$

Let $S \subset W_{\overline{\mathbb{F}}}$ be a very ample non-singular hypersurface whose intersection with each component of $f^{-1}(\xi)$ is irreducible. The geometric generic fiber of $g=\left.f\right|_{S}$ is connected. We choose $S$ so that it is smooth. Write $N_{\xi}\left(W_{\overline{\mathbb{F}}}\right) \subset$ N.S. $\left(W_{\overline{\mathbb{F}}}\right) \otimes \mathbb{Q}$ (respectively $N_{\xi}(S) \subset$ N.S. $\left.(S) \otimes \mathbb{Q}\right)$ for the 
subspace of the Néron-Severi group generated by the components of fibers over $\xi$. The left kernel in the intersection pairing in the top row of

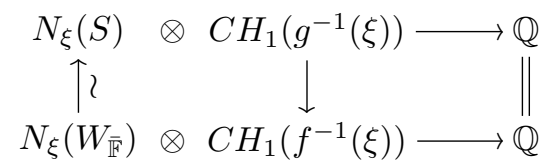

has dimension 1 by the well-known theorem on intersections of components of singular fibers in a fibred surface [9, Proposition 2.6]. Thus $\operatorname{dim}\left(h\left(N_{\xi}\left(W_{\overline{\mathbb{F}}}\right)\right)\right) \geqslant \operatorname{dim}\left(N_{\xi}\left(W_{\overline{\mathbb{F}}}\right)\right)-1=2 m^{2}-1$. Observe that $T_{i} \cdot f^{-1}(\xi) \in C H_{1}\left(f^{-1}(\xi)\right)$ lies in the right kernel of the bottom row of (3.2) since on $W_{\overline{\mathbb{F}}}, T_{i} \cdot f^{-1}(\xi)$ is numerically equivalent to $T_{i} \cdot f^{-1}(x)$ for any $x \in X_{\overline{\mathbb{F}}}$. As the pairing

$$
\operatorname{Span}\left\{S_{i}\right\}_{1 \leqslant i \leqslant 3} \otimes \operatorname{Span}\left\{T_{i} \cdot f^{-1}(\xi)\right\}_{1 \leqslant i \leqslant 3} \longrightarrow \mathbb{Q}
$$

is non-degenerate by $(3.1), \operatorname{rank}(h) \geqslant 2 m^{2}-1+3$. By Lemma $3.8, h$ is surjective.

To complete the proof of Proposition 3.5 we need only show that $\sum_{\xi \in \Xi_{\overline{\mathbb{P}}}} a_{i, \xi} S_{i} \cdot f^{-1}(\xi)$ gives a torsion class in $C H^{2}\left(W_{\overline{\mathbb{F}}}\right)$. Since $T_{i} \cdot \sum_{\xi \in \Xi_{\overline{\mathbb{P}}}} z_{\xi}^{\prime}=0$, it follows that $\sum_{\xi \in \Xi_{\overline{\mathbb{F}}}} a_{i, \xi}=0$. Since $\mathbb{F}$ is finite, $\sum_{\xi \in \Xi_{\overline{\mathbb{F}}}} a_{i, \xi} \xi \in C H^{1}\left(X_{\overline{\mathbb{F}}}\right)_{\text {tors }}$. Applying $f^{*}: C H^{1}\left(X_{\overline{\mathbb{F}}}\right) \rightarrow C H^{1}\left(W_{\overline{\mathbb{F}}}\right)$ and intersecting with $S_{i}$ gives the desired result.

\section{Drinfeld modules and level structures}

The proof of our main theorem requires a detailed understanding of the geometry of the compactification of moduli schemes for rank 2 Drinfeld modules with $\Gamma_{0}(\mathfrak{c})$-level structure. In particular we need information about the singular fibers (cf. Proposition 9.6), Atkin-Lehner automorphisms (cf. Theorem 8.1), the Manin-Drinfeld theorem (cf. Theorem 7.1), and modular parametrizations of elliptic curves (cf. Theorem 10.1).

In this section we recall the notion of Drinfeld module and various notions of level structure on a Drinfeld module. The original source for the material in this section and the next several sections is $[\mathbf{1 0}]$. Additional sources include $[8,16,21,32,18]$.

Let $X$ be a smooth, projective, geometrically irreducible curve over a finite field $\mathbb{F}$ of cardinality $q$ and characteristic $p$. Let $x_{\infty} \in X$ be a closed point. Set $A=H^{0}\left(X-\left\{x_{\infty}\right\}, \mathcal{O}_{X}\right)$. Fix an integer $r>0$. For $a \in A \backslash\{0\}$, define $d_{a} \in \mathbb{Z}$ by the formula $q^{d_{a}}=\#(A / a A)^{r}$. Then

$$
d_{a}=-r \cdot \operatorname{ord}_{x_{\infty}}(a) \cdot\left[\mathbb{F}\left(x_{\infty}\right): \mathbb{F}\right] .
$$

Let $S$ be an $A$-scheme.

Definition 4.1. A Drinfeld $A$-module over $S$ of rank $r$ consists of a group scheme $\mathbb{G} \rightarrow S$ and a ring homomorphism $\Phi: A \rightarrow \operatorname{End}(\mathbb{G}), a \mapsto \Phi_{a}$, satisfying the following conditions.

(a) There is a covering of $S$ by affine open subsets such that for each $U=\operatorname{Spec}(B)$ in the cover, the restriction $\mathbb{G}_{U}$ of $\mathbb{G}$ is isomorphic to the additive group $\mathbb{G}_{a, U}=\operatorname{Spec}(B[x])$.

(b) For any $a \in A \backslash\{0\}$, the resulting diagram

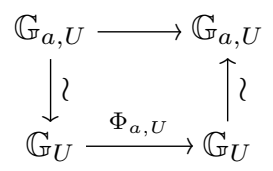

yields an endomorphism of $\mathbb{G}_{a, U}=\operatorname{Spec}(B[x])$, with the property that it is given by an $\mathbb{F}$-linear polynomial $\sum_{n \geqslant 0} b_{n}(a) x^{q^{n}}$ such that $b_{0}: A \rightarrow B$ is the structure morphism to the $A$-algebra $B, b_{d_{a}}(a) \in B$ is a unit, and $b_{n}(a)$ is nilpotent for $n>d_{a}$. 
A morphism, $\lambda: \Phi \rightarrow \Phi^{\prime}$, of Drinfeld $A$-modules over $S$ is a homomorphism of $S$-group schemes satisfying $\lambda \circ \Phi(a)=\Phi^{\prime}(a) \circ \lambda$ for all $a \in A$.

One thinks of a Drinfeld module as a way to describe an $A$-module structure on the group scheme $\mathbb{G}$. For a rank $r$ module, this structure has the property that the $a$-torsion for $a \in$ $A \backslash\{0\}$ is a finite subgroup scheme of rank $d_{a}=\#(A / a A)^{r}$.

We now recall the notion of a Drinfeld level structure $[\mathbf{1 0}, \mathbf{2 0}]$. Let $\mathfrak{b} \subset A$, with $\mathfrak{b} \neq(0)$, be an ideal. Any element $b \in \mathfrak{b}$ defines an endomorphism multiplication by $b$ (written $\Phi_{b}$ ) on a Drinfeld $A$-module $(\mathbb{G}, \Phi)$. Its kernel is denoted $\mathbb{G}[b]$; for $b \neq 0$ this is a subgroup scheme of rank $\#(A / b A)^{r}$. The intersection $\times_{\mathbb{G}} \mathbb{G}[b]$ over all $b \in \mathfrak{b}$ will be written as $\mathbb{G}[\mathfrak{b}]$. A full level $\mathfrak{b}$-structure, also called a $\Gamma(\mathfrak{b})$-structure, on a rank $r$ Drinfeld $A$-module $\mathbb{G}$ over $S$ is a group homomorphism $\varphi:(A / \mathfrak{b})^{r} \rightarrow \mathbb{G}(S)$ such that

$$
\mathbb{G}[\mathfrak{b}]=\sum_{x \in(A / \mathfrak{b})^{r}}[\varphi(x)] .
$$

This is interpreted as an equality of Cartier divisors.

Suppose now that $r=2$. A $\Gamma_{0}(\mathfrak{b})$-structure is defined as a finite flat subgroup scheme $H \subset \mathbb{G}[\mathfrak{b}]$ with an induced action of $A / \mathfrak{b}$. Moreover, $H$ is assumed to be cyclic and of constant rank $\#(A / \mathfrak{b})$. This means that a finite, faithfully flat base change $T \rightarrow S$ exists, and there is a point $P \in\left(\mathbb{G} \times{ }_{S} T\right)(T)$ such that

$$
H \times{ }_{S} T=\sum_{x \in A / \mathfrak{b}}[x P],
$$

interpreted as an equality of Cartier divisors.

\section{Modular varieties for $\Gamma(\mathfrak{b})$-level structure}

In this section we gather together basic facts about Drinfeld modular varieties with full level $\mathfrak{b}$-structure.

Suppose $r>0$ is an integer. Suppose $\mathfrak{b} \subset A$ is a non-zero ideal which is divisible by at least two distinct primes. Consider the functor $\mathcal{M}^{r}(\mathfrak{b})$ which assigns to an $A$-scheme $S$ the set of isomorphism classes of rank $r$ Drinfeld $A$-modules over $S$ equipped with a full level $\mathfrak{b}$-structure.

Theorem 5.1. The functor $\mathcal{M}^{r}(\mathfrak{b})$ is representable by a finite type affine $A$-scheme $M^{r}(\mathfrak{b})$.

Proof. See $[\mathbf{1 0}, 5.3]$ or $[\mathbf{2 1}, 2.5]$ or Saïdi's article [16, p. 24] or [32, 5.3.3].

For each maximal ideal $\mathfrak{m} \subset A$ write $\hat{A}_{\mathfrak{m}}$ for the $\mathfrak{m}$-adic completion of $A$. Let $K$ denote the quotient field of $A, K_{\infty}$ the completion at the place $x_{\infty}, A_{\infty} \subset K_{\infty}$ the valuation ring, $\pi_{\infty} \subset A_{\infty}$ a uniformizer, and $\mathbf{C}$ the completion of the algebraic closure of $K_{\infty}$.

TheOREM 5.2. (i) The scheme $M^{r}(\mathfrak{b})$ is regular of dimension $r$.

(ii) The structure map $\varsigma^{r}(\mathfrak{b}): M^{r}(\mathfrak{b}) \rightarrow \operatorname{Spec}(A)$ is flat and surjective. It is smooth over $\operatorname{Spec}(A) \backslash \operatorname{Spec}(A / \mathfrak{b})$.

(iii) The scheme $M^{r}(\mathfrak{b})$ is irreducible.

(iv) There is a natural left action of the finite group $\operatorname{Pic}(A) \times \operatorname{GL}(r, A / \mathfrak{b}) / \mathbb{F}^{*} \operatorname{Id}$ on $M^{r}(\mathfrak{b})$.

(v) The scheme $M^{1}(\mathfrak{b})$ is isomorphic to the affine scheme with coordinate ring the integral closure of $A$ in the abelian extension $K \subset K(\mathfrak{b})$, which by class field theory corresponds to the following subgroup of the idele class group of $K$ :

$$
K^{*}\left(K_{\infty}^{*} \cdot \prod_{\mathfrak{m} \nmid \mathfrak{b}} \hat{A}_{\mathfrak{m}}^{*} \cdot \prod_{\mathfrak{m} \mid \mathfrak{b}}\left(1+\mathfrak{b} \hat{A}_{\mathfrak{m}}\right)\right) \subset \mathcal{I}_{K} / K^{*}
$$


In particular, $M^{1}(\mathfrak{b})$ is Galois over $\operatorname{Spec}(A)$ with Galois group $\operatorname{Pic}(A) \times \operatorname{GL}(1, A / \mathfrak{b}) / \mathbb{F}^{*} \operatorname{Id}$, and $K(\mathfrak{b})$ is the maximal abelian extension of $K$ totally split at infinity, with conductor $\mathfrak{b}$, in which $\mathbb{F}$ is algebraically closed.

(vi) The structure morphism factors:

$$
\varsigma^{r}(\mathfrak{b}): M^{r}(\mathfrak{b}) \stackrel{\vartheta^{r}(\mathfrak{b})}{\longrightarrow} M^{1}(\mathfrak{b}) \stackrel{\epsilon(\mathfrak{b})}{\longrightarrow} \operatorname{Spec}(A) .
$$

Furthermore, $\vartheta^{r}(\mathfrak{b})$ is compatible with group actions in (iv) and the homomorphism

$$
(\mathrm{Id}, \operatorname{det}): \operatorname{Pic}(A) \times \operatorname{GL}(r, A / \mathfrak{b}) / \mathbb{F}^{*} \operatorname{Id} \longrightarrow \operatorname{Pic}(A) \times \operatorname{GL}(1, A / \mathfrak{b}) / \mathbb{F}^{*} \mathrm{Id} .
$$

Proof. (i) and (ii) See $[\mathbf{1 0}, 5 \mathrm{C} ; \mathbf{2 1}, 3.4]$.

(iii) See $[\mathbf{1 8}, 5.10$ and 5.3.1].

(iv) See $[\mathbf{1 0}, 5 \mathrm{D} ; \mathbf{2 1}, 3.5]$. For definiteness we note that a matrix $g \in \mathrm{GL}(r, A / \mathfrak{b})$ acts on column vectors in $(A / \mathfrak{b})^{r}$ from the left. In the notation of $\S 4$ the action on level structures is by $g * \varphi=\varphi \circ g^{-1}$.

(v) See $[\mathbf{1 0}, \S 8]$ and the article by Rust and Scheja [16, Lecture 4, 6.4-6.6]; compare [16, Lecture $9,2.1]$ where the special case $A=\mathbb{F}_{q}[T]$ is considered.

(vi) In $[\mathbf{1 8}, 5.4]$ van der Heiden gives the argument in the case that $\mathfrak{b}$ is a principal ideal. To treat the general case take the quotient by the group $G$ in $[\mathbf{1 8}, 5.3 .1]$.

As we are primarily concerned with moduli of Drinfeld modules of rank 2, we will generally drop the superscript $r$ from the notation when $r=2$ (for example, $M(\mathfrak{b})=M^{2}(\mathfrak{b})$ ). We now review the (relative) 'compactification' of $M(\mathfrak{b})$.

TheOREm 5.3. (i) There exists an irreducible, regular scheme $\bar{M}(\mathfrak{b})$, proper and flat over $\operatorname{Spec}(A)$, containing $M(\mathfrak{b})$ as an open dense subscheme.

(ii) The group action of Theorem 5.2(iv) extends to a biregular action on $\bar{M}(\mathfrak{b})$.

(iii) The map $\vartheta(\mathfrak{b})$ extends to an equivariant morphism

$$
\bar{\vartheta}(\mathfrak{b}): \bar{M}(\mathfrak{b}) \longrightarrow M^{1}(\mathfrak{b}) .
$$

(iv) The fibers of $\bar{\vartheta}(\mathfrak{b})$ are connected.

(v) The structure morphism $\bar{\varsigma}(\mathfrak{b})$ is smooth on the complement of $\bar{\varsigma}(\mathfrak{b})^{-1}(\operatorname{Spec}(A / \mathfrak{b}))$.

(vi) The scheme $\bar{M}(\mathfrak{b})-M(\mathfrak{b})$ with its reduced scheme structure consists of sections of $\bar{\vartheta}(\mathfrak{b})$.

(vii) The group action on $\bar{M}(\mathfrak{b})-M(\mathfrak{b})$ induced by Theorem 5.2(iv) permutes the irreducible components transitively. The stabilizer of one component may be identified with

$$
\{0\} \times N(\mathfrak{b}), \quad \text { where } N(\mathfrak{b}):=\left(\begin{array}{cc}
(A / \mathfrak{b})^{*} & A / \mathfrak{b} \\
0 & \mathbb{F}_{q}^{*}
\end{array}\right) \subset \mathrm{GL}_{2}(A / \mathfrak{b}) .
$$

Proof. (i) See $[\mathbf{2 1}, 5.3 .5]$.

(ii) See $[\mathbf{1 0}, 9.2]$.

(iii) See $[\mathbf{1 0}, 9.3 ; \mathbf{2 1}, 5.3 .5]$.

(iv) By Zariski's connectedness principle $[\mathbf{2 2}, 8.3 .6$ (ii)], it suffices to show that the generic fiber is geometrically connected. In other words, it suffices to show that $\bar{M}(\mathfrak{b}) \times_{\operatorname{Spec}(A)} \operatorname{Spec}(\mathbf{C})$ has $[K(\mathfrak{b}): K]$ connected components. This is known from the analytic theory of Drinfeld modules [16, Lecture 8, pp. 116, 117].

(v) See $[\mathbf{1 0}, 5 \mathrm{C} ; \mathbf{2 1}, 5.3 .5]$.

(vi) See $[\mathbf{1 8}, 5.9]$ or $[\mathbf{2 1}, 5.3 .5]$.

(vii) See $[\mathbf{2 1}, 4.4,5.1 .10$ and 5.3.5] (and also [18, 5.9.2]).

The irreducible components of $\bar{M}(\mathfrak{b})-M(\mathfrak{b})$ are called cusps, as are their images in quotients of $\bar{M}(\mathfrak{b})$ by subgroups of the group described in Theorem 5.3 (vii). 


\section{Modular varieties for $\Gamma_{0}(\mathfrak{c})$-level structures}

References for this section are $[\mathbf{1 4}]$ and $[\mathbf{3 2}, 4.3]$.

If $\mathfrak{c} \subset A$ is divisible by two distinct primes, we define $M_{0}(\mathfrak{c})$ and $\bar{M}_{0}(\mathfrak{c})$ to be the quotients of $M(\mathfrak{c})$ and $\bar{M}(\mathfrak{c})$, respectively, by the subgroup $B(\mathfrak{c}) \subset \mathrm{GL}(2, A / \mathfrak{c})$ consisting of matrices with a zero in the lower left-hand corner. If $\mathfrak{c}$ fails to be divisible by two distinct primes (for example, $\mathfrak{c}=(1)$ ), let $\mathfrak{b}$ be an ideal prime to $\mathfrak{c}$ such that $\mathfrak{b} \mathfrak{c}$ is divisible by two distinct primes. Define $M_{0}(\mathfrak{c})$ to be the quotient of $M(\mathfrak{b} \mathfrak{c})$ by $\mathrm{GL}(2, A / \mathfrak{b}) B(\mathfrak{c})$ (viewed as a subgroup of $\mathrm{GL}(2, A / \mathfrak{b} \mathfrak{c})$ by the Chinese remainder theorem) and define $\bar{M}_{0}(\mathfrak{c})$ analogously. This definition is independent of the choice of auxiliary ideal $\mathfrak{b}$.

Write $\mathcal{M}_{0}(\mathfrak{c})$ for the functor which assigns to each $A$-scheme $S$ the set of isomorphism classes of Drinfeld modules over $S$ with a $\Gamma_{0}(\mathfrak{c})$-level structure. Finally, write $M^{1}(1)$ for the quotient of $M^{1}(\mathfrak{b})$ by the action of $\mathrm{GL}(1, A / \mathfrak{b}) / \mathbb{F}^{*} I d$. This is independent of the choice of $\mathfrak{b}$.

TheOrem 6.1. (i) The quotient $M_{0}(\mathfrak{c})$ is a coarse moduli scheme for the functor $\mathcal{M}_{0}(\mathfrak{c})$.

(ii) The scheme $M^{1}(1)$ is a coarse moduli scheme for rank 1 Drinfeld modules without level structure.

(iii) The quotient $\bar{M}_{0}(\mathfrak{c})$ is irreducible and normal. It is proper and flat over $\operatorname{Spec}(A)$. It contains $M_{0}(\mathfrak{c})$ as an open dense subset.

(iv) The structure morphism factors:

$$
\bar{\varsigma}_{0}(\mathfrak{c}): \bar{M}_{0}(\mathfrak{c}) \stackrel{\bar{\vartheta}_{0}(\mathfrak{c})}{\longrightarrow} M^{1}(1) \stackrel{\epsilon}{\longrightarrow} \operatorname{Spec}(A) .
$$

(v) The fibers of $\bar{\vartheta}_{0}(\mathfrak{c})$ are connected.

(vi) For $z \in M^{1}(1)$ with $\epsilon(z) \notin \operatorname{Spec}(A / \mathfrak{c})$, the fiber $\bar{\vartheta}_{0}(\mathfrak{c})^{-1}(z)$ is irreducible.

(vii) The connected components of $\bar{M}_{0}(\mathfrak{c}) \backslash M_{0}(\mathfrak{c})$ are sections of $\bar{\vartheta}_{0}(\mathfrak{c})$. They are in bijective correspondence with elements of the set $\operatorname{Pic}(A) \times B(\mathfrak{c}) \backslash \mathrm{GL}(2, A / \mathfrak{c}) / N(\mathfrak{c})$.

Proof. (i) and (ii) See [32, 5.3.5].

(iii) This follows from the analogous properties in the case of full level structure by taking quotients.

(iv) Choose $\mathfrak{b} \subset A$ as above. Now (iv) follows from Theorem 5.3(iii) and Theorem 5.2(vi) by taking the quotient of $\bar{M}(\mathfrak{b} \mathfrak{c})$ by the action of $\operatorname{GL}(2, A / \mathfrak{b}) B(\mathfrak{c})$.

(v) Choose $\mathfrak{b} \subset A$ as above. Let $\mathfrak{z} \in M^{1}(\mathfrak{b} \mathfrak{c})$ lie over $z \in M^{1}(1)$. By Theorem 5.3(iv) the fiber $\bar{\vartheta}(\mathfrak{b} \mathfrak{c})^{-1}(\mathfrak{z})$ is connected. Since det $: \operatorname{GL}(2, A / \mathfrak{b}) B(\mathfrak{c}) \rightarrow \operatorname{GL}(1, A / \mathfrak{b} \mathfrak{c})$ is surjective, the quotient map $\bar{M}(\mathfrak{b} \mathfrak{c}) \rightarrow \bar{M}_{0}(\mathfrak{c})$ sends $\bar{\vartheta}(\mathfrak{b} \mathfrak{c})^{-1}(\mathfrak{z})$ onto $\bar{\vartheta}_{0}(\mathfrak{c})^{-1}(z)$.

(vi) Choose $\mathfrak{b} \subset A$ as above so that $\epsilon(z) \notin \operatorname{Spec}(A / \mathfrak{b} \mathfrak{c})$. Let $\mathfrak{z} \in M^{1}(\mathfrak{b} \mathfrak{c})$ lie over $z$. By Theorem 5.3(iv) and (v) the fiber $\bar{\vartheta}(\mathfrak{b} \mathfrak{c})^{-1}(\mathfrak{z})$ is irreducible. The result follows as in (v).

(vii) The first assertion follows from Theorem 5.3(vi). The second assertion follows from Theorem 5.3(vii) by taking quotients.

\section{The Manin-Drinfeld theorem}

An important result for classical modular curves is the Manin-Drinfeld theorem. Gekeler [15] has proved an analog for Drinfeld modular curves. We need this result in the following form.

Theorem 7.1. For any two cusps $c_{K}, c_{K}^{\prime} \in \bar{M}_{0}(\mathfrak{c})_{K}, c_{K}-c_{K}^{\prime} \in C H^{1}\left(\bar{M}_{0}(\mathfrak{c})_{K}\right)_{\text {tors }}$.

Proof. Gekeler's theorem says that, for any irreducible component of $\bar{M}_{0}(\mathfrak{c})_{\mathbf{C}}$, any degree zero divisor supported on the cusps is torsion in the Chow group. Since the cusps are algebraic over $K$, the statement remains true when $\mathbf{C}$ is replaced by a suitable finite extension $K^{\prime}$ of $K$. 
By Theorem 6.1(iii), $\bar{M}_{0}(\mathfrak{c})_{K}$ is irreducible. By Theorem 6.1(vii), $\operatorname{deg}\left(c_{K}-c_{K}^{\prime}\right)=0$. Thus we may realize a positive multiple of $c_{K}-c_{K}^{\prime}$ as the norm of a degree zero divisor supported on the cusps of an irreducible component of $\bar{M}_{0}(\mathfrak{c})_{K^{\prime}}$. The result follows from $[\mathbf{1 2}, 1.4]$.

\section{Atkin-Lehner automorphisms}

We need a notion for Drinfeld modular curves which is analogous to Atkin-Lehner involutions of elliptic modular curves. The treatment here extends somewhat that in [14].

Henceforth we assume that $\mathfrak{m}, \mathfrak{b}$ and $\mathfrak{c}$ are pairwise coprime ideals in $A$. We say that a rank 2 Drinfeld module has a $\Gamma_{0}(\mathfrak{m}) \Gamma(\mathfrak{b} \mathfrak{c})$-level structure when it has both a $\Gamma_{0}(\mathfrak{m})$ - and a $\Gamma(\mathfrak{b} \mathfrak{c})$-level structure. Suppose that $\mathfrak{b} \mathfrak{c}$ is divisible by two distinct primes (note that here and elsewhere we do not exclude the case $\mathfrak{c}=1)$. The functor rank 2 Drinfeld $A$-modules with a $\Gamma_{0}(\mathfrak{m}) \Gamma(\mathfrak{b} \mathfrak{c})$ level structure is representable $[\mathbf{3 2}, 5.3 .3]$. We denote the corresponding fine moduli space by $M\left(\mathfrak{m}_{0}, \mathfrak{b} \mathfrak{c}\right)$ and compactify it, embedding it in $\bar{M}\left(\mathfrak{m}_{0}, \mathfrak{b c}\right):=\bar{M}(\mathfrak{m} \mathfrak{b} \mathfrak{c}) / B(\mathfrak{m})$.

Suppose given a rank 2 Drinfeld $A$-module $(\mathbb{G}, \Phi)$ equipped with a $\Gamma_{0}(\mathfrak{m})$-structure $H^{\prime}$ and a $\Gamma_{0}(\mathfrak{c})$-structure $H^{\prime \prime}$. Then $H=H^{\prime}+H^{\prime \prime}$ gives a $\Gamma_{0}(\mathfrak{m} \mathfrak{c})$-structure.

TheOREM 8.1. (i) The association

$$
(\mathbb{G}, H) \longrightarrow\left(\mathbb{G} / H^{\prime}, H / H^{\prime}+\mathbb{G}[\mathfrak{m}] / H^{\prime}\right)
$$

gives rise to a natural transformation of functors

$$
w_{\mathfrak{m}}: \mathcal{M}_{0}(\mathfrak{m} \mathfrak{c}) \longrightarrow \mathcal{M}_{0}(\mathfrak{m} \mathfrak{c}) .
$$

(ii) The map $w_{\mathfrak{m}}$ induces a morphism $w_{\mathfrak{m}}: M_{0}(\mathfrak{m c}) \rightarrow M_{0}(\mathfrak{m c})$ of schemes over $\operatorname{Spec}(A)$.

(iii) The map $w_{\mathfrak{m}}$ is an automorphism whose order divides $2 n$, where $n$ is the order of $\mathfrak{m}$ in $\operatorname{Pic}(A)$.

(iv) The map $w_{\mathfrak{m}}$ extends to an automorphism of $\bar{M}_{0}(\mathfrak{m c})$.

Proof. (i) Given a Drinfeld module $(\mathbb{G}, \Phi)$ with $\Gamma_{0}(\mathfrak{m c})$-level structure, taking the quotient by the finite flat group scheme associated to the $\Gamma_{0}(\mathfrak{m})$-level structure gives a group scheme which is locally isomorphic to $\mathbb{G}_{a}[\mathbf{3 2}, 2.3 .2]$. Each $\Phi(a) \in \operatorname{End}(\mathbb{G})$ induces an endomorphism of the quotient. This gives rise to a Drinfeld module on the quotient $[\mathbf{3 2}, 3.2 .4]$ which acquires a level $\Gamma_{0}(\mathfrak{c})$-structure from the image of the $\Gamma_{0}(\mathfrak{c})$-structure on $(\mathbb{G}, \Phi)$ and a $\Gamma_{0}(\mathfrak{m})$-structure from the image of $\mathbb{G}[\mathfrak{m}]$.

(ii) Fix an ideal $\mathfrak{b}$ which is prime to $\mathfrak{m} \mathfrak{c}$ with the property that $\mathfrak{b} \mathfrak{c}$ is divisible by two distinct primes. By an argument analogous to the proof of (i), the association $\mathbb{G} \rightarrow \mathbb{G} / H^{\prime}$ gives a natural transformation from the functor Drinfeld $A$-modules with $\Gamma_{0}(\mathfrak{m}) \Gamma(\mathfrak{b c})$-structure to itself. As this functor is representable, we obtain a morphism of fine moduli spaces which is $\mathrm{GL}(2, A / \mathfrak{b}) B(\mathfrak{c})$-equivariant. Passing to the quotient gives the desired map $w_{\mathfrak{m}}$.

(iii) It suffices to show that $w_{\mathfrak{m}}^{2 n}=\operatorname{Id}$ on $M_{0}(\mathfrak{m c})(\mathbf{C})$. Since $M_{0}(\mathfrak{m c})$ is a coarse moduli scheme, an element of $M_{0}(\mathfrak{m c})(\mathbf{C})$ corresponds to a Drinfeld module with $\Gamma_{0}(\mathfrak{m c})$-level structure over $\mathbf{C}$. By the analytic theory of Drinfeld modules, a Drinfeld module over $\mathbf{C}$ is given by $\mathbf{C} / \Lambda$, where $\Lambda \subset \mathbf{C}$ a discrete subgroup, which is a rank 2 projective $A$-submodule (cf. Lopez's article [16, p. 37]). A $\Gamma_{0}(\mathfrak{m c})$-structure on $\mathbf{C} / \Lambda$ is given by a second projective $A$-submodule $\Lambda^{\prime} \subset \mathbf{C}$ satisfying $(\mathfrak{m c})^{-1} \Lambda \supset \Lambda^{\prime} \supset \Lambda$ and $\Lambda^{\prime} / \Lambda \cong(\mathfrak{m c})^{-1} A / A$ as $A$-modules. In this language the Atkin-Lehner map $w_{\mathfrak{m}}$ sends a pair $\left(\Lambda, \Lambda^{\prime}\right)$ to $\left(\Lambda^{\prime}, \mathfrak{m}^{-1} \Lambda\right)$. Iterating $2 n$ times gives $\left(\mathfrak{m}^{-n} \Lambda, \mathfrak{m}^{-n} \Lambda^{\prime}\right)$, which is isomorphic to the original pair since $\mathfrak{m}^{n}$ is principal.

(iv) See $[\mathbf{1 0}, 9.2]$.

Forgetting the $\Gamma_{0}(\mathfrak{m})$-level structure gives a natural transformation $\gamma: \mathcal{M}_{0}(\mathfrak{m} \mathfrak{c}) \rightarrow \mathcal{M}_{0}(\mathfrak{c})$. 
LEMma 8.2. (i) The natural transformation $\gamma$ gives rise to a finite surjective morphism $\gamma: \bar{M}_{0}(\mathfrak{m} \mathfrak{c}) \rightarrow \bar{M}_{0}(\mathfrak{c})$. For each cusp $\bar{c} \subset \bar{M}_{0}(\mathfrak{c}), \gamma^{-1}(\bar{c})$ is supported on cusps.

(ii) When $\mathfrak{m}$ is a maximal ideal, $\operatorname{deg}(\gamma)=1+\#(A / \mathfrak{m})$.

Proof. (i) Forgetting the $\Gamma_{0}(\mathfrak{m})$-level structure gives a natural transformation from the functor rank 2 Drinfeld $A$-modules with $\Gamma_{0}(\mathfrak{m}) \Gamma(\mathfrak{b c})$-level structure to rank 2 Drinfeld $A$-modules with $\Gamma(\mathfrak{b c})$-level structure. This gives a morphism of fine moduli spaces,

$$
\tilde{\gamma}: M\left(\mathfrak{m}_{0}, \mathfrak{b c}\right) \longrightarrow M(\mathfrak{b} \mathfrak{c}),
$$

which induces $\gamma: M_{0}(\mathfrak{m} \mathfrak{c}) \rightarrow M_{0}(\mathfrak{c})$ by taking the quotient by the natural $\operatorname{GL}(2, \mathfrak{b}) B(\mathfrak{c})$-action. One deduces from $[\mathbf{2 1}, 3.4 .1$ (ii)] that both $\tilde{\gamma}$ and $\gamma$ are finite and surjective. They extend to finite morphisms of the compactifications by $[\mathbf{1 0}, 9.2]$. The final assertion in (i) is clear.

(ii) We need only count the number of points in the inverse image of a sufficiently general geometric point. The $\mathfrak{m}$-torsion in a Drinfeld $A$-module over $\mathbf{C}$ is isomorphic to a 2-dimensional $A / \mathfrak{m}$-vector space. Now $\Gamma_{0}(\mathfrak{m})$-structures correspond to 1-dimensional subspaces. There are $1+\#(A / \mathfrak{m})$ of these.

\section{Geometry of cusps and special fibers}

Henceforth $\mathfrak{m} \subset A$ will denote a maximal ideal prime to $\mathfrak{c}$. This section contains the technical results about the cusps of $\bar{M}_{0}(\mathfrak{m c})$ and the fiber $\bar{\zeta}_{0}(\mathfrak{m c})^{-1}(\mathfrak{m})$ which are needed for the construction of torsion cycles in the proof of the main theorem. As in the previous section we make frequent use of an auxillary ideal $\mathfrak{b}$ prime to $\mathfrak{m} \mathfrak{c}$ and such that $\mathfrak{b} \mathfrak{c}$ is divisible by two distinct primes.

For an integral $A$-scheme $M, M_{K}$ denotes the generic fiber and $K(M)$ denotes the field of rational functions.

Lemma 9.1. For any cusp $c_{K} \in \bar{M}(\mathfrak{b} \mathfrak{c})_{K}$, the fiber $\tilde{\gamma}_{K}^{-1}\left(c_{K}\right) \subset \bar{M}\left(\mathfrak{m}_{0}, \mathfrak{b} \mathfrak{c}\right)_{K}$ consists of two cusps. The map $\tilde{\gamma}_{K}$ is unramified at one cusp and has ramification degree $\#(A / \mathfrak{m})$ at the other. The same statements hold for $\gamma_{K}: \bar{M}_{0}(\mathfrak{m c})_{K} \rightarrow \bar{M}_{0}(\mathfrak{c})_{K}$.

Proof. By Theorem 5.3(vii) the cusps of $\bar{M}(\mathfrak{b} \mathfrak{c})_{K}$ are parametrized by cosets

$$
\operatorname{Pic}(A) \times \operatorname{GL}(2, A / \mathfrak{m b} \mathfrak{c}) / N(\mathfrak{m} \mathfrak{b} \mathfrak{c}) .
$$

Thus cusps of $\bar{M}\left(\mathfrak{m}_{0}, \mathfrak{b c}\right)_{K}$ are parametrized by double cosets

$$
\operatorname{Pic}(A) \times B(\mathfrak{m}) \backslash \mathrm{GL}(2, A / \mathfrak{m} \mathfrak{b} \mathfrak{c}) / N(\mathfrak{m b} \mathfrak{c}) .
$$

By the Chinese remainder theorem cusps of $\bar{M}\left(\mathfrak{m}_{0}, \mathfrak{b} \mathfrak{c}\right)$ lying over a given cusp of $\bar{M}(\mathfrak{b} \mathfrak{c})$ are parametrized by

$$
B(\mathfrak{m}) \backslash \mathrm{GL}(2, A / \mathfrak{m}) / N(\mathfrak{m})=B(\mathfrak{m}) \backslash \mathrm{GL}(2, A / \mathfrak{m}) / B(\mathfrak{m}) .
$$

This is the classical Bruhat decomposition of $\mathrm{GL}(2, A / \mathfrak{m})$ and consists of two elements. The $B(\mathfrak{m})$-orbit, $B(\mathfrak{m}) / N(\mathfrak{m}) \subset \mathrm{GL}(2, A / \mathfrak{m}) / N(\mathfrak{m})$, corresponds to cusps of $\bar{M}(\mathfrak{b} \mathfrak{c m})_{K}$ over $c_{K}$ whose decomposition field in the extension $K(M(\mathfrak{b} \mathfrak{c})) \subset K(M(\mathfrak{m b} \mathfrak{c}))$ is $K(M(\mathfrak{m b c}))^{B(\mathfrak{m})}$. The corresponding cusp of $\bar{M}\left(\mathfrak{m}_{0}, \mathfrak{b c}\right)_{K}$ is unramified. The same argument as was used in Lemma 8.2(ii) shows that $\tilde{\gamma}_{K}$ has degree $1+\#(A / \mathfrak{m})$. Thus the second cusp in $\tilde{\gamma}_{K}^{-1}\left(c_{K}\right)$ has ramification degree $\#(A / \mathfrak{m})$. The corresponding result for $\gamma_{K}$ follows by taking the quotient by $\operatorname{GL}(2, A / \mathfrak{b}) B(\mathfrak{c})$.

We next study the fiber $\bar{\zeta}_{0}(\mathfrak{m c})^{-1}(\mathfrak{m})(\mathrm{cf} .[\mathbf{1 4}, \S 5])$. In preparation we recall a few basic facts about automorphisms of Drinfeld modules. 
Lemma 9.2. (i) Let $\Phi$ be a rank 2 Drinfeld $A$-module. Then $\mathbb{F}^{*} \simeq A^{*} \simeq \operatorname{Aut}(\Phi)$ or $\mathbf{F}^{*} \simeq \operatorname{Aut}(\Phi)$ where $\mathbf{F}$ is a quadratic extension of $\mathbb{F}$.

(ii) For each maximal ideal $\mathfrak{m} \subset A$ there exist only finitely many isomorphism classes of rank 2 Drinfeld $A$-modules $\Phi$ over the algebraic closure of $A / \mathfrak{m}$ for which Aut $(\Phi) \not 飞 \mathbb{F}^{*}$.

Proof. (i) See $[\mathbf{1 6}, 2.11$, p. 73].

(ii) With the notation used in (i), suppose that $\operatorname{Aut}(\Phi)=\mathbf{F}^{*} \not \mathbb{F}^{*}=A^{*}$. Put $B:=\mathbf{F} \otimes_{\mathbb{F}} A$. The Drinfeld $A$-module $\Phi$ naturally yields a Drinfeld $B$-module of rank 1 . Vice versa, any Drinfeld $B$-module of rank 1 yields by restriction to $A$ a rank 2 Drinfeld $A$-module with automorphism group $\mathbf{F}^{*}$. Since there are only finitely many isomorphism classes of such Drinfeld $B$-modules, the result follows.

Set $k=A / \mathfrak{m}$ and use the subscript ${ }_{k}$ to denote base change with respect to $A \rightarrow A / \mathfrak{m}$ (for example, $\left.\bar{M}_{0}(\mathfrak{c})_{k}:=\bar{M}_{0}(\mathfrak{c}) \otimes_{A} A / \mathfrak{m}\right)$. Then $\bar{M}_{0}(\mathfrak{c})_{k}$ is a fiber of the quotient variety $\bar{M}(\mathfrak{b} \mathfrak{c}) / \operatorname{GL}(2, A / \mathfrak{b}) B(\mathfrak{c})$. The next lemma compares this with the quotient of the fiber $\bar{M}(\mathfrak{b} \mathfrak{c})_{k}$. Write $\breve{M}_{0}(\mathfrak{c})_{k}$ for the normalization of $\bar{M}_{0}(\mathfrak{c})_{k}$ at the cusps. A recent result of Taelman [32, Theorem 5.5.2] implies that the normal variety $\bar{M}_{0}(\mathfrak{c})$ is regular along the cusps. It follows that $\breve{M}_{0}(\mathfrak{c})_{k} \simeq \bar{M}_{0}(\mathfrak{c})_{k}$. However, we have not used this here; instead, we apply Lemma 9.4 below which seems to be of independent interest.

Lemma 9.3. $\quad \breve{M}_{0}(\mathfrak{c})_{k} \simeq \bar{M}(\mathfrak{b} \mathfrak{c})_{k} / \mathrm{GL}(2, A / \mathfrak{b}) B(\mathfrak{c})$.

Proof. Note that $\mathrm{GL}(2, A / \mathfrak{b}) B(\mathfrak{c}) / \mathbb{F}^{*}$ Id acts on $\bar{M}(\mathfrak{b} \mathfrak{c})$ and $\bar{M}(\mathfrak{b} \mathfrak{c})_{k}$, by Lemma $9.2(\mathrm{i})$. There are open dense subsets in each of these varieties where the stabilizer at any closed point is trivial, by Lemma 9.2(ii). Since $\bar{M}(\mathfrak{b c})$ is smooth over $\operatorname{Spec}(A)-\operatorname{Spec}(A / \mathfrak{b c})$ (by Theorem $5.3(\mathrm{v})$ ), $\bar{M}(\mathfrak{b} \mathfrak{c})_{k}$ is a smooth curve and so is $\bar{M}(\mathfrak{b} \mathfrak{c})_{k} / \mathrm{GL}(2, A / \mathfrak{b}) B(\mathfrak{c})$. Thus it suffices to show that the natural morphism $M(\mathfrak{b} \mathfrak{c})_{k} / \mathrm{GL}(2, A / \mathfrak{b}) B(\mathfrak{c}) \rightarrow M_{0}(\mathfrak{c})_{k}$ is an isomorphism.

Observe that the stabilizer of $\mathrm{GL}(2, A / \mathfrak{b}) B(\mathfrak{c})$ at every closed point of $M(\mathfrak{b} \mathfrak{c})$ has order prime to $p$ by Lemma 9.2(i). Thus the lemma is a consequence of the following result.

Lemma 9.4. Let $f: M \rightarrow C$ be a smooth morphism of varieties in characteristic $p$ with target a smooth curve. Let $G$ be a finite group which acts with stabilizers of order prime to $p$ on $M$. Assume that $f$ is $G$-equivariant when $G$ acts trivially on $C$. Let $c \in C$ be a closed point such that $G$ acts with trivial stabilizers on a dense open subset of $f^{-1}(c)$. Write $\bar{f}: M / G \rightarrow C$ for the obvious map. Then the tautological dominant morphism $f^{-1}(c) / G \rightarrow \bar{f}^{-1}(c)$ is an isomorphism.

Proof. Let $H$ be the stabilizer of a closed point $m \in M$. Write $\bar{m} \in M / H$ for the image of $m$. Let $S$ be the semi-local ring of $M$ at the orbit $G m$. Then $S^{G}$ is a local ring. Let $t \in S^{G}$ be the image of a uniformizing parameter on $C$ at $c$. Consider the following commutative diagram with injective vertical maps:

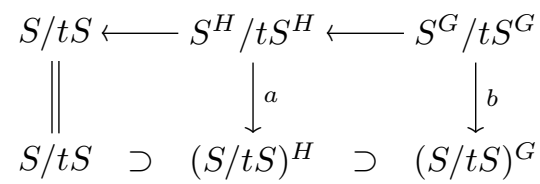

We must show that $b$ is an isomorphism. The map $a$ is an isomorphism since $p \nmid|H|$, so $H^{1}(H, t S)=0$. Smoothness of $f$ implies that $S / t S$ is non-singular, so $(S / t S)^{H}$ and $(S / t S)^{G}$ are normal. Hence $S^{H} / t S^{H}$ is also normal. The localization of $S^{H}$ at $\bar{m}$ is etale over $S^{G}[\mathbf{1 7}$, V.2.2]. Since etaleness is preserved by base change, the localization of $S^{H} / t S^{H}$ at $\bar{m}$ is etale 
over $S^{G} / t S^{G}$. Now $S^{G} / t S^{G}$ is normal since $S^{H} / t S^{H}$ is (see [24, I.3.17]). Since $S$ is integral over $S^{G}, S / t S$ is integral over $S^{G} / t S^{G}$ and $b$ is an integral extension. Since $(S / t S)^{G}$ is normal and local, it is an integral domain.

To show that $b$ is an isomorphism, it suffices to show that the map induced by $b$ on fraction fields is an isomorphism. This calculation may be done at any nearby point on $f^{-1}(c) / G$. Thus we may assume that $H=1$. Now $S$ (respectively $S / t S$ ) is finite and etale over $S^{G}$ (respectively $(S / t S)^{G}$ ). Hence $S$ (respectively $S / t S$ ) is free of rank $|G|$ as an $S^{G}$ (respectively $\left.(S / t S)^{G}\right)$ module. On the other hand, applying $\bigotimes_{S^{G}} S^{G} / t S^{G}$ shows that $S / t S$ is free of rank $|G|$ as an $S^{G} / t S^{G}$-module. Now the inclusion of fraction fields induced by $b$ is an isomorphism since the total quotient ring of $S / t S$ has dimension $|G|$ when viewed as a vector space over either field.

This completes the proof of Lemma 9.3.

Corollary 9.5. The map

$$
\varsigma_{0}(\mathfrak{c}): M_{0}(\mathfrak{c}) \longrightarrow \operatorname{Spec}(A)
$$

is smooth on the complement of $\varsigma_{0}(\mathfrak{c})^{-1}(A / \mathfrak{c})$.

Proof. We note that $\varsigma_{0}(\mathfrak{c})$ is flat by Theorem 6.1(iii), the closed fibers are non-singular curves by Lemma 9.3, and the residue fields of closed points in $\operatorname{Spec}(A)$ are perfect.

Define $d_{\mathfrak{m}}$ by $q^{d_{\mathfrak{m}}}=\# k$. A Drinfeld $A$-module over a scheme $S$ is said to have characteristic $\mathfrak{m}$ if the structure map factors, $S \rightarrow \operatorname{Spec}(A / \mathfrak{m}) \rightarrow \operatorname{Spec}(A)$.

Proposition 9.6. (i) There is a canonical map, $i_{F}: \breve{M}_{0}(\mathfrak{c})_{k} \rightarrow \bar{M}_{0}(\mathfrak{m c})_{k}$, such that the induced map $\gamma_{k} \circ i_{F}$ is the normalization of $\bar{M}_{0}(\mathfrak{c})_{k}$.

(ii) The composition $\gamma_{k} \circ w_{\mathfrak{m}} \circ i_{F}: \breve{M}_{0}(\mathfrak{c})_{k} \rightarrow \bar{M}_{0}(\mathfrak{c})_{k}$ is the Frobenius morphism relative to $k$ composed with the normalization.

(iii) The map

$$
\breve{M}_{0}(\mathfrak{c})_{k} \coprod \breve{M}_{0}(\mathfrak{c})_{k} \stackrel{\left(i_{F}, w_{\mathfrak{m}} \circ i_{F}\right)}{\longrightarrow} \bar{M}_{0}(\mathfrak{m} \mathfrak{c})_{k}
$$

is surjective.

(iv) A cusp $c \subset \bar{M}_{0}(\mathfrak{m c})$ intersects the image of $i_{F}$ if and only if $c_{K}$ is not a ramification point of $\gamma_{K}: \bar{M}_{0}(\mathfrak{m c})_{K} \rightarrow \bar{M}_{0}(\mathfrak{c})_{K}$.

(v) If the cusp $c_{K} \in \bar{M}_{0}(\mathfrak{m c})_{K}$ is unramified under $\gamma_{K}$, then the cusp $w_{\mathfrak{m}}\left(c_{K}\right)$ is ramified.

Proof. (i) Let $\mathfrak{b}$ be an auxiliary ideal as above. For a Drinfeld $A$-module over a scheme $S$ in characteristic $\mathfrak{m}$ with full level $\mathfrak{b} \mathfrak{c}$-structure $(\mathbb{G}, \Phi)$, the line bundle underlying $\mathbb{G}$ is trivialized by the choice of a section $\beta_{x_{0}}$ of the $\mathfrak{b} \mathfrak{c}$-torsion subgroup. Such a Drinfeld module may be viewed as a homomorphism $\Phi: A \rightarrow \mathcal{O}_{S}(S)\{\tau\}$, where multiplication in the skew polynomial ring is determined by $b^{q} \tau=\tau b$. Conjugation in this ring by $F=\tau^{d_{\mathfrak{m}}}$ amounts to raising the coefficients of polynomials in $\tau$ to the $q^{d_{\mathfrak{m}}}$ th power. Now $a \mapsto F \circ \Phi(a) \circ F^{-1}$ is a Drinfeld $A$-module over $S$, since the constant term in each skew polynomial $\Phi(a)$ is fixed by conjugating by $F$. Thus $F$ gives a morphism of Drinfeld $A$-modules over $S, \Phi \mapsto F \Phi F^{-1}$. Clearly $\operatorname{Ker}(F)$ is a $\Gamma_{0}(\mathfrak{m})$-level structure on $\Phi$. Thus we have a natural transformation from the functor Drinfeld $A$-modules in characteristic $\mathfrak{m}$ with $\Gamma(\mathfrak{b} \mathfrak{c})$-level structure to the functor Drinfeld $A$-modules in characteristic $\mathfrak{m}$ with $\Gamma_{0}(\mathfrak{m}) \Gamma(\mathfrak{b} \mathfrak{c})$-level structure. Both of these functors are representable, so the natural transformation corresponds to a morphism between fine moduli spaces. On the smooth curve $M(\mathfrak{b} \mathfrak{c})_{k}$ this map separates points and tangent vectors and is thus an embedding. It extends to a morphism $\tilde{i}_{F}: \bar{M}(\mathfrak{b c})_{k} \rightarrow \bar{M}\left(\mathfrak{m}_{0}, \mathfrak{b c}\right)_{k}$, since $\bar{M}(\mathfrak{b c})$, and hence $\bar{M}(\mathfrak{b} \mathfrak{c})_{k}$, is non-singular. 
Taking the quotient by $\operatorname{GL}(2, A / \mathfrak{b}) B(\mathfrak{c})$ gives a morphism

$$
i_{F}: \breve{M}_{0}(\mathfrak{c})_{k} \longrightarrow \bar{M}\left(\mathfrak{m}_{0}, \mathfrak{b c}\right)_{k} / \mathrm{GL}(2, A / \mathfrak{b}) B(\mathfrak{c}) \longrightarrow \bar{M}_{0}(\mathfrak{m} \mathfrak{c})_{k}
$$

with the property that the composition, $\gamma_{k} \circ i_{F}: \breve{M}_{0}(\mathfrak{c})_{k} \rightarrow \bar{M}_{0}(\mathfrak{c})_{k}$, induces the identity on the open dense subscheme $M_{0}(\mathfrak{c})_{k}$.

(ii) With notation as in part (i), consider the natural transformation $\Phi \mapsto F \Phi F^{-1}$. The second Drinfeld $A$-module acquires a $\Gamma(\mathfrak{b} \mathfrak{c})$-structure by applying $F$ to the $\Gamma(\mathfrak{b} \mathfrak{c})$-structure on $\Phi$. The fine moduli space $M(\mathfrak{b} \mathfrak{c})_{k}$ has a standard description as a subspace of the affine space whose coordinates correspond to coefficients of skew polynomials $\Phi(a)$ and to $\mathfrak{b} c$-torsion sections $[21,2.5]$. The endomorphism $M(\mathfrak{b c})_{k} \rightarrow M(\mathfrak{b c})_{k}$, corresponding to the natural transformation just described, raises all these coordinates to the $q^{d_{\mathfrak{m}}}$-power and is thus Frobenius relative to $k$. This endomorphism descends to $\gamma_{k} \circ w_{\mathfrak{m}} \circ i_{F}: M_{0}(\mathfrak{c})_{k} \rightarrow M_{0}(\mathfrak{c})_{k}$.

(iii) It suffices to show that the image is Zariski dense. Away from the finite set of isomorphism classes of supersingular Drinfeld $A$-modules in characteristic $\mathfrak{m}[\mathbf{1 6}$, p.81], a Drinfeld $A$-module of characteristic $\mathfrak{m}$ over an algebraically closed field has exactly two $\Gamma_{0}(\mathfrak{m})$-structures $[\mathbf{3 2}, 4.3 .2]$.

(iv) The composite $\tilde{\gamma}_{k} \circ \tilde{i}_{F}: M(\mathfrak{b c c})_{k} \rightarrow M(\mathfrak{b} \mathfrak{c})_{k}$ is the identity, while the composite $\tilde{\gamma}_{k} \circ w_{\mathfrak{m}} \circ i_{F}$ is purely inseparable of degree $\#(A / \mathfrak{m})$ by (ii). As noted in the proof of (i), $\tilde{i}_{F}$ extends to a morphism $\tilde{i}_{F}: \bar{M}(\mathfrak{b c})_{k} \rightarrow \bar{M}\left(\mathfrak{m}_{0}, \mathfrak{b} \mathfrak{c}\right)_{k}$ which must be an embedding since $\tilde{\gamma}_{k} \circ \tilde{i}_{F}=$ Id. It is clear that $\tilde{i}_{F}\left(\bar{M}(\mathfrak{b} \mathfrak{c})_{k}\right)$ cannot meet any cusp of $\bar{M}\left(\mathfrak{m}_{0}, \mathfrak{b} \mathfrak{c}\right)$ where $\tilde{\gamma}$ is ramified. Similarly, the embedded curve $w_{\mathfrak{m}} \circ \tilde{i}_{F}\left(\bar{M}(\mathfrak{b} \mathfrak{c})_{k}\right) \subset \bar{M}\left(\mathfrak{m}_{0}, \mathfrak{b c}\right)$ cannot meet any cusp where $\tilde{\gamma}$ is unramified. Now the result follows by taking the quotient by $\operatorname{GL}(2, A / \mathfrak{b}) B(\mathfrak{c})$ and applying (iii).

(v) This follows from the proof of (iv).

\section{Modular parametrizations of elliptic surfaces}

Let $Y_{K}$ be an elliptic curve over $K$ with neutral element $e$. The curve $Y_{K}$ may be regarded as the generic fiber of a unique relatively minimal elliptic surface $\pi: Y \rightarrow X$ with section $\bar{e}$ containing $e$. Assume that $Y_{K}$ has split multiplicative reduction at $x_{\infty}$. The conductor of $Y_{K}$ is a divisor on $X$ which is uniquely expressible as a product of an ideal $\mathfrak{c} \subset A$ with $x_{\infty}$. The complement of the support of the conductor, $\dot{X} \subset X$, is the locus over which $\pi$ is smooth. Write $\dot{Y}, \dot{W}, \dot{\varsigma}_{0}(\mathfrak{c}), \dot{M}_{0}(\mathfrak{c}), \dot{M}^{1}(1)$ and $\dot{\vartheta}_{0}(\mathfrak{c})$ for the base changes of $Y, W, \bar{\varsigma}_{0}(\mathfrak{c}), \bar{M}_{0}(\mathfrak{c}), M^{1}(1)$ and $\bar{\vartheta}_{0}(\mathfrak{c})$ with respect to this inclusion.

TheOREm 10.1. (i) Let $c_{K} \in \bar{M}_{0}(\mathfrak{c})_{K}$ be a cusp. There is a non-constant morphism of $K$-curves $\rho_{K}: \bar{M}_{0}(\mathfrak{c})_{K} \rightarrow Y_{K}$ sending $c$ to $e$.

(ii) The morphism $\rho_{K}$ extends to a finite morphism $\dot{\rho}: \dot{M}_{0}(\mathfrak{c}) \rightarrow \dot{Y}$.

Proof. (i) Let $l \neq p$ be a prime. Drinfeld's great theorem yields a $\operatorname{Gal}(\bar{K} / K)$-submodule of $H^{1}\left(\bar{M}_{0}(\mathfrak{c})_{\bar{K}}, \mathbb{Q}_{l}\right)$ which is isomorphic to $H^{1}\left(Y_{\bar{K}}, \mathbb{Q}_{l}\right)[\mathbf{1 0}, \S 11]$. The Tate conjecture for $\bar{M}_{0}(\mathfrak{c})_{K} \times Y_{K}$ gives a divisor on the product which induces an isomorphism on Galois modules [33, Theorem 4; 37]. By adding and subtracting divisors of the form $\bar{M}_{0}(\mathfrak{c})_{K} \times e$ and $m \times Y_{K}$ and by moving within a rational equivalence class, one may arrange that the divisor is a graph of a morphism $\rho_{0}$. Set $L=K\left(M^{1}(1)\right)$. From the commutative diagram

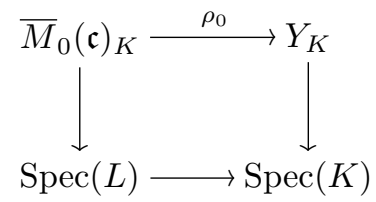


we deduce that $\rho_{0}$ factors:

$$
\bar{M}_{0}(\mathfrak{c})_{K} \stackrel{\rho_{1}}{\longrightarrow} Y_{L} \stackrel{\rho_{2}}{\longrightarrow} Y_{K} .
$$

The image of $c$ is a degree 1 point on $Y_{L}$. Write $\tau: Y_{L} \rightarrow Y_{L}$ for translation by minus the image of $c$. Now $\rho_{K}:=\rho_{2} \circ \tau \circ \rho_{1}$ gives a map with the required properties.

(ii) Choose an ideal $\mathfrak{b}$ prime to $\mathfrak{c}$ such that $\mathfrak{b} \mathfrak{c}$ is divisible by two distinct primes. Set $Y^{\prime}=Y \times_{X} M^{1}(1)$. Set $\ddot{X}=\dot{X}-\operatorname{Supp}(\mathfrak{b})$ and write $\ddot{M}(\mathfrak{b} \mathfrak{c}), \ddot{M}_{0}(\mathfrak{c}), \ddot{M}^{1}(1)$ and $\ddot{Y}^{\prime}$ for, respectively, the base changes of $\bar{M}(\mathfrak{b} \mathfrak{c}), \bar{M}_{0}(\mathfrak{c}), M^{1}(1)$ and $Y^{\prime}$ with respect to the inclusion $\ddot{X} \rightarrow X$. Since $\ddot{M}_{0}(\mathfrak{c})$ is a quotient of $\ddot{M}(\mathfrak{b} \mathfrak{c})$, by (i) there is a rational map

$$
\tilde{\rho}: \ddot{M}(\mathfrak{b} \mathfrak{c}) \longrightarrow \ddot{M}_{0}(\mathfrak{c}) \stackrel{\rho_{1}}{-\rightarrow} \ddot{Y}^{\prime}
$$

of schemes over $\ddot{M}^{1}(1)$. Now $\tilde{\rho}$ may be made into a morphism by blowing up points of indeterminancy. Since $\ddot{M}(\mathfrak{b c})$ is non-singular, by Theorem 5.3(i) any point of indeterminancy would lead to a rational curve in a fiber of $\ddot{Y}^{\prime} \rightarrow \ddot{M}^{1}(1)$. Since no such rational curves exist, $\tilde{\rho}$ is a morphism. The fiber components of the natural map $\ddot{M}(\mathfrak{b c}) \rightarrow \ddot{M}^{1}(1)$ are permuted transitively by $\operatorname{GL}(2, A / \mathfrak{b}) B(\mathfrak{c})$, since $\ddot{\vartheta}_{0}(\mathfrak{c}): \ddot{M}_{0}(\mathfrak{c}) \rightarrow \ddot{M}^{1}(1)$ has irreducible fibers by Theorem $6.1(\mathrm{vi})$. By $(10.2), \tilde{\rho}$ is $\mathrm{GL}(2, A / \mathfrak{b}) B(\mathfrak{c})$-equivariant. Thus it does not contract any fiber component of $\ddot{M}(\mathfrak{b c})$ to a point. Since $\tilde{\rho}$ is proper, it is finite.

The verification that the rational map $\rho_{1}$ is a finite morphism may be done locally on an open affine space, $\operatorname{Spec}(R) \subset \ddot{Y}^{\prime}$ with inverse image $\operatorname{Spec}(S) \subset \ddot{M}(\mathfrak{b c})$ : since $R$ is contained in both $S$ and the $\operatorname{GL}(2, A / \mathfrak{b}) B(\mathfrak{c})$-invariants of the rational functions on $\ddot{M}(\mathfrak{b} \mathfrak{c})$, it is contained in the $\operatorname{GL}(2, A / \mathfrak{b}) B(\mathfrak{c})$-invariants of $S$, which gives an affine open subscheme of $\ddot{M}_{0}(\mathfrak{b} \mathfrak{c})$. Since $S$ is a Noetherian finite $R$-module, so are the invariants. This shows that $\rho_{1}$ in (10.2) is a finite morphism.

Since $\dot{X}$ is covered by open subsets of the form $\dot{X}-\operatorname{Supp}(\mathfrak{b})$ with $\mathfrak{b}$ as above, the rational map $\rho_{1}$ extends to a finite morphism $\dot{M}_{0}(\mathfrak{c}) \rightarrow \dot{Y}^{\prime}$. Composing with $\dot{Y}^{\prime} \rightarrow \dot{Y}$ gives a finite morphism $\dot{\rho}: \dot{M}_{0}(\mathfrak{c}) \rightarrow \dot{Y}$.

\section{Proof of the main theorem}

We keep the notation of previous sections. In particular, $\mathbb{F}$ is a finite field of characteristic $p, X$ is a smooth, projective, geometrically irreducible curve over $\mathbb{F}, \pi: Y \rightarrow X$ is a relatively minimal, semi-stable, non-isotrivial, elliptic surface with section, and $\dot{X} \subset X$ is the locus over which $\pi$ is smooth. Set $\dot{W}=\dot{Y} \times_{\dot{X}} \dot{Y}$ and write $\dot{f}: \dot{W} \rightarrow \dot{X}$ for the tautological map. Let $i_{\bar{x}}: f^{-1}(\bar{x}) \rightarrow W_{\overline{\mathbb{F}}}$ denote the inclusion of a fiber above a CM point $\bar{x} \in X_{\overline{\mathbb{F}}}$.

Theorem 0.2 will follow easily from the following result.

TheOREM 11.1. The image of $i_{\bar{x} *}: C H^{1}\left(f^{-1}(\bar{x})\right) \rightarrow C H^{2}\left(\dot{W}_{\overline{\mathbb{F}}}\right)$ is a torsion group.

Proof. Observe that $C H_{\text {hom }}^{1}\left(f^{-1}(\bar{x})\right)$ is a torsion group since it is isomorphic to the $\overline{\mathbb{F}}$ points of the abelian variety $\operatorname{Pic}^{0}\left(f^{-1}(\bar{x})\right)$. With notation as in $\S 3$, the Néron-Severi group of $f^{-1}(\bar{x})$ is generated by $T_{i} \cdot f^{-1}(\bar{x})$, for $1 \leqslant i \leqslant 3$, and a complex multiplication cycle. Since $\bar{x}$ gives a torsion class in $C H^{1}\left(\dot{X}_{\overline{\mathbb{F}}}\right), f^{-1}(\bar{x}) \in C H^{1}\left(\dot{W}_{\overline{\mathbb{F}}}\right)_{\text {tors }}$ and $T_{i} \cdot f^{-1}(\bar{x}) \in C H^{2}\left(\dot{W}_{\overline{\mathbb{F}}}\right)_{\text {tors }}$. Thus to complete the proof of Theorem 11.1, it suffices to show that a single 1-cycle supported in $f^{-1}(\bar{x})$, whose class in the Néron-Severi group $N^{1}\left(f^{-1}(\bar{x})\right)$ does not lie in the subgroup $N_{0}^{1}\left(f^{-1}(\bar{x})\right)=\operatorname{Span}\left\{T_{i} \cdot f^{-1}(\bar{x})\right\}_{1 \leqslant i \leqslant 3}$, is torsion in $C H^{2}\left(\dot{W}_{\overline{\mathbb{F}}}\right)$.

It is at this point that the theory of Drinfeld modular curves enters. Fix a point $x_{\infty} \in X-\dot{X}$. After replacing $\mathbb{F}$ by a finite extension (also denoted $\mathbb{F}$ ) we may assume that $x_{\infty}$ is a degree 1 point and that $Y$ has split multiplicative reduction at $x_{\infty}$. We may similarly arrange that the point $x \in \dot{X}$, which is the image of $\bar{x}$, is a degree 1 point. Let $A=H^{0}\left(X-\left\{x_{\infty}\right\}, \mathcal{O}_{X}\right)$, 
and write $\mathfrak{m} \subset A$ for the maximal ideal corresponding to $x$ and $\mathfrak{c} \subset A$ for the conductor of $Y_{K}$ divided by $x_{\infty}$.

Consider the composition of finite morphisms

$$
\breve{M}_{0}(\mathfrak{c})_{k} \stackrel{i_{F}}{\longrightarrow} \dot{M}_{0}(\mathfrak{m c}) \stackrel{\left(\gamma, \gamma \circ w_{\mathfrak{m}}\right)}{\longrightarrow} \dot{M}_{0}(\mathfrak{c}) \times_{\dot{X}} \dot{M}_{0}(\mathfrak{c}) \stackrel{\dot{\rho} \times \dot{\rho}}{\longrightarrow} \dot{W} .
$$

By Proposition 9.6(i) and (ii), the image of $\left(\gamma, \gamma \circ w_{\mathfrak{m}}\right)_{k} \circ i_{F}$ is the graph of the $k$-linear Frobenius morphism in $\dot{M}_{0}(\mathfrak{c})_{k}^{2}$. Applying $(\dot{\rho}, \dot{\rho})_{*}$ to this graph gives $\operatorname{deg}(\dot{\rho}) \Gamma$, where $\Gamma \subset Y_{k}^{2}$ is the graph of Frobenius on the elliptic curve $Y_{k}$. By Theorem 6.1(iv), the irreducible components of $\dot{M}_{0}(\mathfrak{c})_{k}$ are the fibers $\dot{\vartheta}_{0}(\mathfrak{c})^{-1}\left(z_{i}\right)$, where $\left\{z_{1}, \ldots, z_{s}\right\}=\epsilon^{-1}(\mathfrak{m}) \subset \dot{M}^{1}(1)$. Let $C_{i}$ be the closure of $i_{F}\left(\vartheta_{0}(\mathfrak{c})^{-1}\left(z_{i}\right)\right)$. Then

$$
(\dot{\rho}, \dot{\rho})_{*} \circ\left(\gamma, \gamma \circ w_{\mathfrak{m}}\right)_{*} C_{i}=e_{i} \Gamma \quad \text { with } e_{i}>0 .
$$

The connected components of $\dot{M}_{0}(\mathfrak{m c})_{k}$ are the fibers $\dot{\vartheta}_{0}(\mathfrak{m c})^{-1}\left(z_{i}\right)$. Besides $C_{i}$ this fiber has a second irreducible component $C_{i}^{\prime}$, by Proposition 9.6(iii).

Fix a cusp $\dot{c} \subset \dot{M}_{0}(\mathfrak{m c})$ which is not contained in the ramification locus of

$$
\dot{\gamma}: \dot{M}_{0}(\mathfrak{m c}) \longrightarrow \dot{M}_{0}(\mathfrak{c}) .
$$

Such cusps exist by Lemma 9.1. Write $K\left(\dot{M}_{0}(\mathfrak{m c})\right)$ and $L=K\left(M^{1}(1)\right)$ for the fields of rational functions on $\dot{M}_{0}(\mathfrak{m c})$ and $M^{1}(1)$.

Lemma 11.2. There are $N_{0}, \ldots, N_{s} \in \mathbb{N}$ and $\beta \in K\left(\dot{M}_{0}(\mathfrak{m c})\right)^{*}$ such that

$$
\operatorname{div}(\beta)=N_{0}\left(\dot{c}-w_{\mathfrak{m}} \dot{c}\right)+\sum_{i=1}^{s} N_{i} C_{i} \in \operatorname{Div}\left(\dot{M}_{0}(\mathfrak{m} \mathfrak{c})\right) .
$$

Proof. By the Manin-Drinfeld theorem, Theorem 7.1, there exist $\beta^{\prime} \in K\left(\dot{M}_{0}(\mathfrak{m c})\right)$ and $N^{\prime} \in \mathbb{N}$ such that, on the generic fiber,

$$
\operatorname{div}\left(\beta^{\prime}\right)=N^{\prime}\left(c_{K}-w_{\mathfrak{m}} c_{K}\right) \in \operatorname{Div}\left(\dot{M}_{0}(\mathfrak{m c})_{K}\right) .
$$

Thus there is a divisor $D^{\prime}$ supported on closed fibers of $\dot{\vartheta}_{0}(\mathfrak{m c})$ so that

$$
\operatorname{div}\left(\beta^{\prime}\right)=N^{\prime}\left(\dot{c}-w_{\mathfrak{m}} \dot{c}\right)+D^{\prime} \in \operatorname{Div}\left(\dot{M}_{0}(\mathfrak{m} \mathfrak{c})\right) .
$$

Every closed fiber of $\dot{\vartheta}_{0}(\mathfrak{m c})$ may be regarded as a torsion element of $C H^{1}\left(\dot{M}_{0}(\mathfrak{m c})\right)$, since every closed point of the affine curve $\dot{M}^{1}(1)$ may be regarded as a torsion element of $C H^{1}\left(\dot{M}^{1}(1)\right)$. At the expense of replacing $N^{\prime}$ by a multiple and replacing $\beta^{\prime}$ by a power of itself times an element of $L^{*}$, we may arrange that $D^{\prime}$ is supported on the reducible fibers of $\dot{\vartheta}_{0}(\mathfrak{m c})$.

The only reducible fibers of $\dot{\vartheta}_{0}(\mathfrak{m c})$ are those that lie above $\mathfrak{m}$, by Theorem 6.1 (vi). Thus we may write

$$
D^{\prime}=\sum_{i=1}^{s} n_{i} C_{i}+n_{i}^{\prime} C_{i}^{\prime} .
$$

Let $\nu_{i}$ denote the order of $z_{i}$ in $\operatorname{Pic}\left(M^{1}(1)\right)$. Choose $\ell_{i} \in L^{*}$ with $\operatorname{div}\left(\ell_{i}\right)=-\nu_{i} z_{i}$. By (11.4) and (11.5), $C_{i}^{\prime}$ appears with zero multiplicity in the prime decomposition of the divisor $\operatorname{div}\left(\ell_{i}^{n_{i}^{\prime}}\left(\beta^{\prime}\right)^{\nu_{i}}\right)$. By iterating this process, we produce a function $\beta$ of the form $\prod_{i=1}^{s} \ell_{i}^{M_{i}}\left(\beta^{\prime}\right)^{M}$, with $\operatorname{div}(\beta)=N_{0}\left(\dot{c}-w_{\mathfrak{m}} \dot{c}\right)+\sum_{i=1}^{s} N_{i} C_{i}$, where $N_{0} \in \mathbb{N}$. Intersecting with $C_{i}$ gives

$$
0=\operatorname{deg}\left(\left.\operatorname{div}(\beta)\right|_{C_{i}}\right)=N_{0} \dot{c} \cdot C_{i}+N_{i} C_{i} \cdot C_{i}=N_{0} \dot{c} \cdot C_{i}-N_{i} C_{i}^{\prime} \cdot C_{i},
$$

since $w_{\mathfrak{m}} \dot{c} \cdot C_{i}=0$ by Proposition 9.6(iv) and (v), $C_{j} \cdot C_{i}=0$ for $i \neq j$ and $\left(C_{i}+C_{i}^{\prime}\right) \cdot C_{i}=0$ because $C_{i}+C_{i}^{\prime}$ is the fiber $\dot{\vartheta}_{0}(\mathfrak{m c})^{-1}\left(z_{i}\right)$. The intersection theory being used here is that for a quotient variety as described in [12,8.3.12 and 16.1.13]. This is permissible since $\dot{M}_{0}(\mathfrak{m c})$ is the quotient of a non-singular variety by the action of a finite group (cf. Section 6). Note that 
$\dot{M}_{0}(\mathfrak{m c})$ may be singular at points of $C_{i} \cap C_{i}^{\prime}$ (see $[\mathbf{1 4}, 5.8]$ ). As $\dot{c} \cdot C_{i}>0$ and $C_{i}^{\prime} \cdot C_{i}>0$, we have $N_{i}>0$.

Since $\dot{\rho}$ sends one cusp of $\dot{M}_{0}(\mathfrak{c})$ to the zero section of $\dot{Y}$, it sends other cusps to torsion sections of $\dot{Y}$. Choose $m \in \mathbb{N}$ such that multiplication by $m, \mathbf{m} \in \operatorname{End}(\dot{Y} / \dot{X})$, sends each of these torsion sections to the zero section. Observe that

$$
\mathbf{g}:=(\mathbf{m} \circ \dot{\rho}, \mathbf{m} \circ \dot{\rho}) \circ\left(\gamma, \gamma \circ w_{\mathfrak{m}}\right): \dot{M}_{0}(\mathfrak{m c}) \longrightarrow \dot{W}
$$

maps both cusps $\dot{c}$ and $w_{\mathfrak{m}} \dot{c}$ to the zero section of $\dot{W}$. Thus applying $\mathbf{g}_{*}$ to (11.3) yields

$$
0=\mathbf{g}_{*}(\operatorname{div}(\beta))=m^{2}\left(\sum_{i=1}^{s} N_{i} e_{i}\right) \Gamma \in C H^{2}(\dot{W}) .
$$

Now $\Gamma$, and hence the positive multiple of $\Gamma$ which appears in (11.6), does not lie in the subspace $N_{0}^{1}\left(f^{-1}(\bar{x})\right)$ of $N^{1}\left(f^{-1}(\bar{x})\right)$. Applying the pullback map $C H^{2}(\dot{W}) \rightarrow C H^{2}\left(\dot{W}_{\overline{\mathbb{F}}}\right)$ proves Theorem 11.1.

Proof of Theorem 0.2. In the notation of Proposition 3.5, Theorem 11.1 implies that $\tilde{j}^{*}\left(C H_{\mathrm{CM}}^{2}\left(W_{\overline{\mathbb{F}}}\right)\right)$ is a torsion group. Since $C H_{\mathrm{CM}}^{2}\left(W_{\overline{\mathbb{F}}}\right) \subset C H_{\mathrm{hom}}^{2}\left(W_{\overline{\mathbb{F}}}\right)$, Proposition 3.5 implies that $C H_{\mathrm{CM}}^{2}\left(W_{\overline{\mathbb{F}}}\right)$ is a torsion group. Now Theorem 0.2 follows from Propositions 3.3 and 3.4, Lemma 2.1, and Proposition 1.1.

\section{References}

1. S. AbHyankar, Resolution of singularities of embedded algebraic surfaces (Academic Press, New York, 1966).

2. A. BeILInson, 'Height pairing between algebraic cycles', Current trends in arithmetical algebraic geometry, Arcata, CA, 1985 (ed. K. A. Ribet), Contemporary Mathematics 67 (American Mathematical Society, Providence, RI, 1987) 1-24.

3. S. BLOCH, 'Torsion algebraic cycles and a theorem of Roitman', Compositio Math. 39 (1979) 107-127.

4. S. BLOCH, 'Algebraic cycles and values of $L$-functions', J. reine angew. Math. 350 (1984) 94-108.

5. J.-L. Colliot-ThÉLÈne, 'Cycles algébriques de torsion et $K$-théorie algébrique', Arithmetic algebraic geometry, Trento, 1991 (ed. E. Ballico), Lecture Notes in Mathematics 1553 (Springer, New York, 1993) 1-49.

6. J.-L. Colliot-Thélène, J.-J. Sansuc and C. Soulé, 'Torsion dans le groupe de Chow de codimension deux', Duke Math. J. 50 (1983) 763-801.

7. P. Deligne, 'Théorie de Hodge II', Inst. Hautes Études Sci. Publ. Math. 40 (1972) 5-57.

8. P. Deligne and D. Husemoller, 'Survey of Drinfeld modules', Current trends in arithmetical algebraic geometry, Arcata, CA, 1985 (ed. K. A. Ribet), Contemporary Mathematics 67 (American Mathematical Society, Providence, RI, 1987) 25-91.

9. M. Deschamps, 'Réduction semi-stable', Séminaire sur les pinceaux de courbes de genre au moins deux (ed. L. Szprio), Astérisque 86 (Société Mathématique de France, Paris, 1981) 1-34.

10. V. G. DrinfEL'D, 'Elliptic modules', Math. USSR Sbornik 23 (1976) 561-592.

11. M. FlaCH, 'A finiteness theorem for the symmetric square of an elliptic curve', Invent. Math. 109 (1992) 307-327.

12. W. Fulton, Intersection theory (Springer, New-York, 1984).

13. O. GabBer, 'Sur la torsion dans la cohomologie $l$-adique d'une varietié', $C$. $R$. Acad. Sci. Paris Sér. I Math. 297 (1983) 179-183.

14. E.-U. GeKeler, 'Über Drinfeldsche Modulkurven vom Hecke-Typ', Compositio Math. 57 (1986) 219-236.

15. E.-U. GeKeler, 'A note on the finiteness of certain cuspidal divisor class groups', Israel J. Math. 118 (2000) 357-368.

16. E.-U. Gekeler, M. van der Put, M. Reversat and J. Van Geel (eds), Drinfeld modules, modular schemes and applications (World Scientific, Singapore, 1997).

17. A. Grothendieck, Revêtements étales et groupe fondamental (SGA 1), Lecture Notes in Mathematics 224 (Springer, New York, 1971).

18. G.-J. van DER Heiden, 'Weil pairing and the Drinfeld modular curve', PhD thesis, Groningen, 2003, http://irs.ub.rug.nl/ppn/25493871X; parts published as 'Factoring polynomials over finite fields using Drinfeld modules', Math. Comp. 73 (2004) 317-322, 'Local-global problem for Drinfeld modules', J. Number Theory 104 (2004) 193-209, 'Weil pairing for Drinfeld modules', Monatsh. Math. 143 (2004) 115-143, and 'Drinfeld modular curve and Weil pairing', J. Algebra 299 (2006) 374-418). 
19. U. Jannsen, Mixed motives and algebraic K-theory, Lecture Notes in Mathematics 1400 (Springer, New York, 1990).

20. N. M. Katz and B. Mazur, Arithmetic moduli of elliptic curves, Annals of Mathematics Studies 108 (Princeton University Press, 1985).

21. T. Lenmkuhl, 'Compactification of the Drinfeld modular surfaces', Habilitationsschrift, Göttingen, 2000.

22. Q. LiU, Algebraic geometry and arithmetic curves (Oxford University Press, 2002).

23. S. Mildenhall, 'Cycles in a product of elliptic curves and a group analogous to the class group', Duke Math J. 67 (1992) 387-406.

24. J. Milne, Étale cohomology (Princeton University Press, 1980).

25. C. Schoen, 'On the computation of the cycle class map for nullhomologous cycles over the algebraic closure of a finite field', Ann. Sci. École Norm. Sup. (4) 28 (1995) 1-50.

26. C. Schoen, 'On the image of the $l$-adic Abel-Jacobi map for a variety over the algebraic closure of a finite field', J. Amer. Math. Soc. 12 (1999) 795-838.

27. C. Schoen, 'Specialization of the torsion subgroup of the Chow group', Math. Z. 252 (2006) 11-17.

28. C. Schoen, 'Torsion in the cohomology of fiber products of elliptic surfaces', Preprint, Duke University, 2002.

29. C. Schoen, 'Divisor class groups of certain normal crossing surfaces', Preprint, Duke University, 2002.

30. J. H. Silverman, The arithmetic of elliptic curves (Springer, New York, 1986).

31. C. Soulé, 'Groupes de Chow et K-théorie de variétés sur un corps fini', Math. Ann. 268 (1984) 317-345.

32. L. TAElman, 'Drinfeld modular curves have many points', Master's Thesis, Groningen, 2002 , arXiv:math.AG/0602157.

33. J. TATE, 'Endomorphisms of abelian varieties over finite fields', Invent. Math. 2 (1966) 134-144.

34. J. TATE, 'Relations between $K_{2}$ and Galois cohomology', Invent. Math. 36 (1976) 257-274.

35. B. Totaro, 'Torsion algebraic cycles and complex cobordism', J. Amer. Math. Soc. 10 (1997) 467-493.

36. C. Weibel, An introduction to homological algebra (Cambridge University Press, 1994).

37. Y. ZARHIN, 'Endomorphisms of abelian varieties over fields of finite characteristic', Math. USSR Izvestija 9 (1975) 255-260.

Chad Schoen

Department of Mathematics

Duke University

Box 90320

Durham, NC 27708-0320

USA

schoen@math.duke.edu
Jaap Top

IWI

University of Groningen

P.O. Box 800

9700 AV Groningen

The Netherlands

top@math.rug.nl 